\title{
Optoelectronic Characterization of Transmission Lines and Waveguides by Terahertz Time-Domain Spectroscopy
}

\author{
Daniel R. Grischkowsky, Fellow, IEEE
}

Invited Paper

\begin{abstract}
An overview is presented of an experimental search for an ultrawide-band transmission channel with low dispersion and loss. Such a terahertz (THz) interconnect will soon be required by the insatiable demand for higher speed devices and wider bandwidth communication. Starting with the early optoelectronic generation and detection of single-mode, subpicosecond electrical pulses on coplanar transmission lines, their complete characterization by THz time-domain spectroscopy (THz-TDS) is described. The consequent discovery of phase-coherent Cherenkov radiation in the form of an electromagnetic shock wave from these propagating electrical pulses is discussed together with its dominant role in the large measured propagation loss of these pulses. Various techniques to reduce this radiation are presented. The importance of dielectric materials characterization is explained and illustrated by THz-TDS measurements of high $T_{c}$ substrates. Newly obtained THz waveguide results are presented and compared to the performance of coplanar transmission lines.
\end{abstract}

Index Terms-Copolanar transmission lines, frequency-domain analysis, millimeter-wave measurement, millimeter-wave propagation, millimeter-wave spectroscopy, millimeter-wave waveguide, optoelectronics, time-domain analysis, time-domain measurements, transmission lines, waveguides.

\section{INTRODUCTION}

$\mathbf{T}$ HE IMPORTANCE of propagation of picosecond (ps) or subpicosecond (subps) pulses on micrometer- or submicrometer-sized wiring has forced the consideration of guided-wave propagation effects even on the single electronic chip level. Phenomena previously considered only by the microwave community in the frequency domain and at gigahertz frequencies are now becoming manifest in the ps time-domain at terahertz $(\mathrm{THz})$ frequencies. The basic characteristics of propagation of ps/subps pulses on lithographically defined transmission lines need to be fully understood with respect to realizable design parameters. For example, it has been shown that the loss properties are very different for pulses

Manuscript received August 16, 2000. This work was supported in part by the National Science Foundation and the Army Research Office.

The author is with the School of Electrical and Computer Engineering and the Center for Laser and Photonics Research, Oklahoma State University, Stillwater, OK 74078 USA.

Publisher Item Identifier S 1077-260X(00)11543-6. propagating as electric dipoles or electric quadrupoles. The same expectation holds for cross talk as well. Modal considerations are very important to the excitation and measurement of transmission lines. The excitation of nonpropagating modes can lead to severe pulse broadening. Due to the increase in line resistance as the wire cross sections are reduced, the bandwidth of transmission lines markedly decreases as the wire dimensions are reduced below $1 \mu \mathrm{m}$. This situation has spurred the investigation of normal superconducting lines, high $T_{c}$ superconducting lines, and cryogenically cooled crystalline normal metal lines. The bandwidth of transmission lines is also dependent upon the transparency of the dielectric substrate to the propagating frequencies, and such data are often lacking.

Optoelectronic techniques combining ultrafast laser pulses, photoconductive switching [1], electrooptic sampling [2], and very large scale integration (VLSI) lithography have enabled the generation and measurement of subps electrical pulses propagating as a single-mode excitation on coplanar transmission lines [1]-[5]. Besides their many applications for device characterization [1], [2], [6], these pulses can also be used to characterize the transmission line itself, by the powerful method of THz time-domain spectroscopy (THz-TDS) [7]-[9].

The advent of optoelectronic sources and receivers of subps pulses of freely propagating $\mathrm{THz}$ electromagnetic radiation has generated much interest in the propagation properties of these pulses. For example, all of the pulse propagation problems cited above for transmission lines disappear for the quasi-optical approach using freely propagating $\mathrm{THz}$ beams for which subps $\mathrm{THz}$ pulses can propagate hundreds of centimeters without distortion [10]. An associated technical problem has been the efficient coupling between freely propagating $\mathrm{THz}$ waves and the guided-wave propagation of such $\mathrm{THz}$ radiation. Recently, efficient broadband coupling of freely propagating pulses of $\mathrm{THz}$ electromagnetic radiation into circular and rectangular metal waveguides and single-crystal sapphire fibers has been demonstrated [11]-[13]. Single-mode coupling and propagation were achieved, even though the spectral bandwidth overlapped as many as 25 additional modes. Such waveguide propagation has already demonstrated much larger bandwidths with approximately one-tenth the loss compared to the lithographically defined planar transmission lines. 
(a)

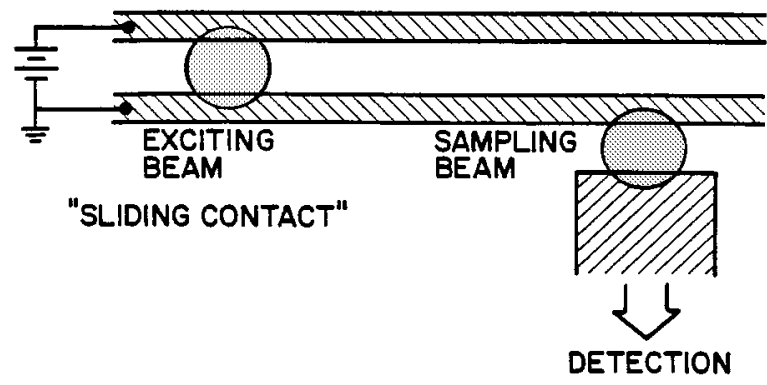

(b)

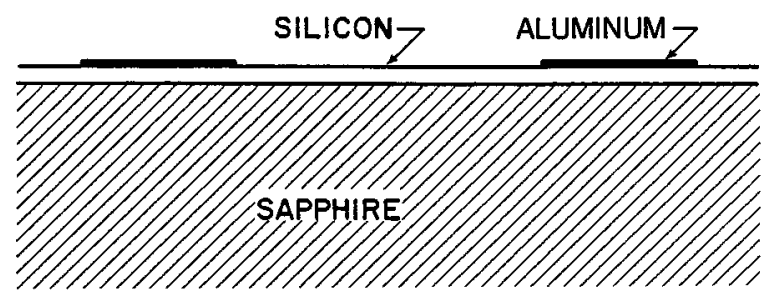

Fig. 1. (a) Experimental geometry. (b) Cross section of SOS wafer.

\section{GENERATION OF SINGLE-MODE UlTRASHORT ELECTRICAL PULSES ON TRANSMISSION LINES}

An early optoelectronic method of generation and detection of single-mode ultrashort electrical pulses is illustrated in Fig. 1 [4], [5]. This technique is still used today and will be described in some detail. A coplanar transmission line composed of aluminum metal lines lithographically fabricated on a silicon-onsapphire (SOS) substrate is shown schematically. Typically, the lines are several centimeters long, $5 \mu \mathrm{m}$ wide, $0.5 \mu \mathrm{m}$ thick, and separated from each other by $10 \mu \mathrm{m}$, giving a design impedance of $100 \Omega$. A commercial SOS wafer, shown in cross section, is composed of a $0.5-\mu \mathrm{m}$-thick layer of silicon on a sapphire substrate. The wafer was heavily implanted with oxygen to ensure a short carrier lifetime. Originally, this electrical arrangement was driven by 70-fs ultrashort optical laser pulses from a compensated, colliding-pulse, passively mode-locked dye laser producing pulses at a $100-\mathrm{MHz}$ repetition rate. At the present time, shorter and more powerful pulses from Ti : Sapphire lasers are used. The laser beam is divided into two beams, indicated as the exciting and sampling beams in the figure, where the sampling pulses have a variable delay with respect to the excitation pulses. During operation, the excitation pulses short the transmission line by producing carriers in the silicon. These carriers are trapped in less than $600 \mathrm{fs}$ [14]. This transient shorting of the line produces two approximately 0.5 -ps electrical pulses which propagate as single-mode excitations in opposite directions down the transmission line. The single-mode feature is due to the micrometer-sized dimensions of the coplanar line and to the "sliding contact" method of excitation [4], [5] which matches the TEM mode of the transmission line. The generated and propagated pulses traveling in one direction are measured by the sampling pulses at the detection gap. As the pulse traverses the line adjacent to the detection gap, a voltage appears across this gap. If this gap is then connected to the line by the sampling pulse, current will flow during the time of this connection. The amount of collected charge will be proportional to the voltage across the gap during the sampling interval. Consequently, the collected charge is measured versus the com-
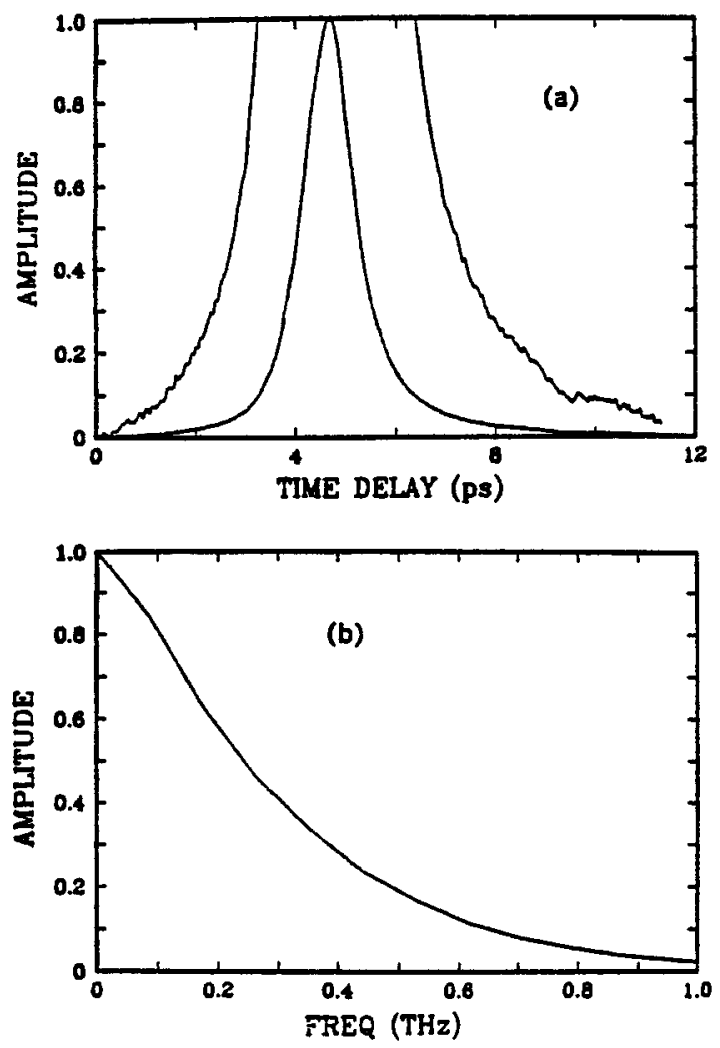

Fig. 2. (a) Measured ultrashort electrical pulse (lower trace), $10 \times$ expanded scale (upper trace). (b) Amplitude spectrum of pulse.

puter-controlled relative time delay between the excitation and sampling pulses.

For the generated pulse shown in Fig. 2(a), the excitation site was very close to the sampling gap so that propagation effects were absent. This measurement illustrates the exceptionally clean pulses and high signal-to-noise ratios which can be obtained with this technique. The pulse was measured with a single scan of the optical delay line and had an amplitude of about $10 \mathrm{mV}$. In the figure, we show the same pulse on a $10 \times$ expanded scale, where our signal-to-noise ratio of 500:1 (in a single scan) can be seen. Fig. 2(b) shows the Fourier transform (amplitude spectrum) of this pulse to illustrate the bandwidth available.

\section{TRANSMISSION LINE THEORY}

We will now discuss some general aspects of transmission line theory in the quasi-static limit for which the wavelengths involved are large compared to the transverse dimensions of the line [15]. For this case, a two-line transmission line has a single propagating TEM mode; the electric field pattern of this mode (shown in Fig. 3) is the same as that for the static case. Any pulse propagating on this line can be described mathematically as a Fourier sum of single-frequency components all with this same frequency-independent TEM field pattern.

Consideration of the sliding contact excitation site shows that, to first order, charge is simply transferred from one line to the other creating a symmetrical field distribution with respect to the two lines. During the excitation process, a current flow is induced between the lines. Localized charge accumulations of 


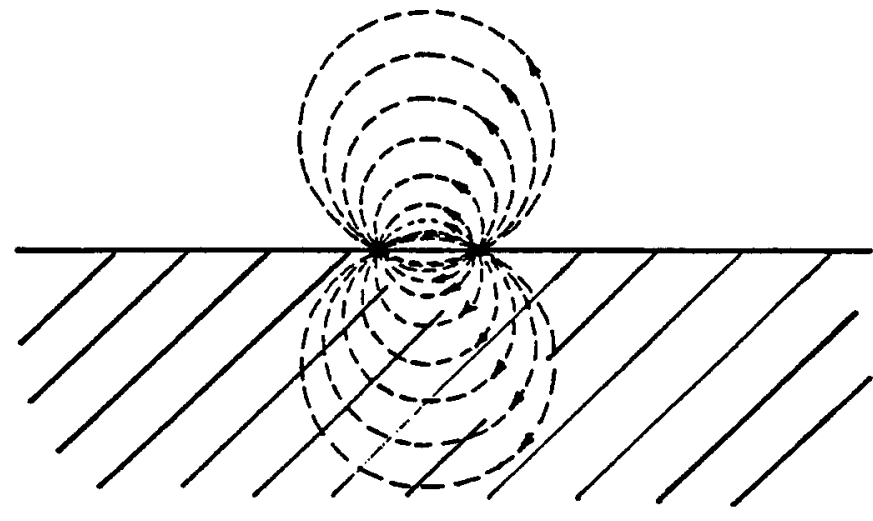

Fig. 3. Electrical field lines for the propagating TEM mode.

opposite sign build up on the segments of the two metal lines under the laser excitation spot, creating a dipolar field distribution similar to that illustrated in Fig. 3 and thereby, efficiently coupling the sliding contact excitation to the TEM mode.

A most important feature of a coplanar transmission line (of negligible thickness) on an infinite dielectric half-space is that for a constant voltage between the two conducting lines the electric field lines are the same as for the lines immersed in free space [15]. This result is due to the geometric symmetry with respect to the dielectric boundary and is a consequence of the fact that no electric field lines cross this boundary.

For this simple situation, it has been shown that the phase velocity is given by [15]

$$
v_{p}=c(2 /(\varepsilon(\omega)+1))^{1 / 2} .
$$

If we define the effective index $n_{e}=c / v_{p}$, the expression for the group velocity becomes

$$
v_{g}=c /\left[n_{e}+\omega\left(d n_{e} / d \omega\right)\right] .
$$

For a relatively nondispersive dielectric, e.g., sapphire, $v_{p} \cong v_{g}$. The power absorption coefficient $\alpha$ for the transmission line can be written as the sum of the absorption due to the metal lines $\alpha_{m l}$ and that due to the dielectric $\alpha_{d l}$

$$
\alpha=\alpha_{m l}+\alpha_{d l}
$$

The dielectric loss of the line can be written in the very general form for guided waves [12]

$$
\alpha_{d l}=f_{f} \alpha_{d} v_{g d} / v_{g}
$$

where the filling factor $f_{f}$ is defined as the ratio of the energy per unit length in the dielectric to the total energy per unit length on the coplanar line, $\alpha_{d}$ is the power absorption coefficient in the bulk dielectric, and $v_{g d}$ is the group velocity in the dielectric. For the simple case of the coplanar waveguide with the same field pattern in the air and in the dielectric, $f_{f}$ is given by $f_{f}=$ $\varepsilon /(1+\varepsilon) ; v_{g d}=c / \varepsilon^{1 / 2}$, and $\alpha_{d l}$ is evaluated to be

$$
\alpha_{d l}=\alpha_{d}[\varepsilon /(2+2 \varepsilon)]^{1 / 2} .
$$

\section{THz-TDS CHARACTERIZATION OF DIELECTRICS AND TRANSMISSION LINES}

The powerful technique of THz-TDS is especially appropriate for characterizing dielectrics, ultrawide-band transmission lines, and waveguides [7]-[9]. With this technique, two electromagnetic pulse shapes are measured, the input pulse and the propagated pulse which has changed shape due to its passage through the sample under study. Consequently, via Fourier analysis of the input and propagated pulses, the frequency-dependent absorption and dispersion of the sample can be obtained. The useful frequency range of the method is determined by the initial pulse duration and the time resolution of the detection process. Therefore, with each reduction in the width of the generated electromagnetic pulse, and/or the time resolution of detection, there is a corresponding increase in the available frequency range. Clearly, THz-TDS can be used with both guided-wave and freely propagating $\mathrm{THz}$ pulses.

The subps electrical pulses generated on coplanar transmission lines can be used for THz-TDS characterization of the transmission line itself. This is due to two important features. Firstly, the useful bandwidth of the pulses extends up to $1 \mathrm{THz}$, and secondly, the pulses propagate as a single mode of excitation on the transmission line. As the ultrashort electrical pulse propagates down the transmission line, its pulse shape will change due only to the frequency-dependent electrical and magnetic properties of the transmission line, e.g., the metal of the line, the dielectric substrate, and radiation processes. Consequently, via THz-TDS, the absorption and dispersion versus frequency of the line can be obtained [16].

\section{A. Dielectric Characterization}

The goal to have a high-speed coplanar transmission line capable of transmitting a subps electrical pulse a distance of the order of $10 \mathrm{~mm}$ places severe requirements on the $\mathrm{THz}$ transparency of the underlying dielectric substrate. This requirement has generated new interest in the $\mathrm{THz}$ characterization of such dielectric materials [7]. For a specific example, assume that it is desired to propagate a subps signal with a $2-\mathrm{THz}$ bandwidth and that the power loss at $2 \mathrm{THz}$ should be less than 0.5 after 10 $\mathrm{mm}$ of travel on the transmission line. At room temperature the absorption of sapphire, fused silica, semi-insulating GaAs and $\mathrm{MgO}$ is too high. Crystalline quartz is quite acceptable. Plastics such as high-density polyethylene and TPX would also be acceptable. High-resistivity float-zone, intrinsic silicon is practically transparent with a power absorption coefficient of less than $0.05 \mathrm{~cm}^{-1}$ for frequencies below and including $2 \mathrm{THz}$.

These considerations become especially important with respect to the realization of a high-bandwidth coplanar transmission line fabricated out of the new high $T_{c}$ superconducting materials. The THz-TDS characterization at both room temperature and $85 \mathrm{~K}$ of three dielectric substrates used with high $T_{c}$ coplanar transmission lines, namely, magnesium oxide, yttria-stabilized zirconia (YSZ), and lanthanum aluminate, provided limits on the realizable bandwidths obtainable [17]. In particular, YSZ shows in Fig. 4(a) a very large absorption with a power absorption coefficient at $85 \mathrm{~K}$ approaching $80 \mathrm{~cm}^{-1}$ at $1 \mathrm{THz}$. For YSZ, the transmission full-width at half-maximum 

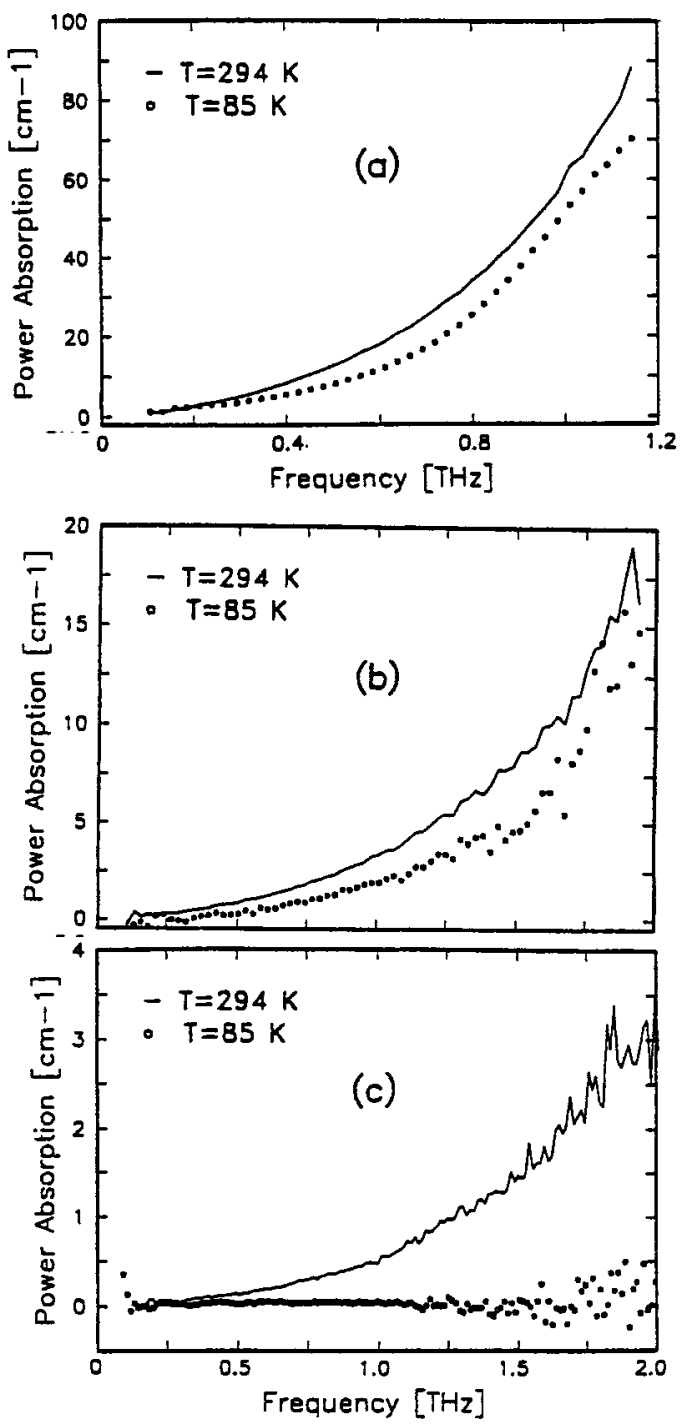

Fig. 4. Power absorption measurements at room temperature (solid lines) and at $85 \mathrm{~K}$ (circles). (a) YSZ. (b) Lanthanum aluminate. (c) $\mathrm{MgO}$.

(FWHM) bandwidth through a 10-mm coplanar line would be less than $200 \mathrm{GHz}$. Lanthanum aluminate is significantly better, showing in Fig. 4(b) a power absorption coefficient at $85 \mathrm{~K}$ of approximately $2 \mathrm{~cm}^{-1}$ at $1 \mathrm{THz}$, giving a transmission bandwidth through a $10-\mathrm{mm}$ coplanar line of $0.7 \mathrm{THz}$. In contrast, $\mathrm{MgO}$ appears in Fig. 4(c) to be quite transparent to $\mathrm{THz}$ radiation at $85 \mathrm{~K}$ and would have a transmission bandwidth through a 10-mm coplanar line well above $2 \mathrm{THz}$.

\section{B. Ordinary Metal Transmission Lines}

We now describe an early application of THz-TDS to characterize a transmission line [16], based on measurements of subps electrical pulses propagating on a coplanar transmission line [4]. The 20-mm-long transmission line was composed of three 5- $\mu \mathrm{m}$ -wide lines separated by $10 \mu \mathrm{m}$, lithographically fabricated on an ion-implanted SOS wafer. The sliding contact method of excitation generated the optically sampled voltage pulses shown in Fig. 5 [4]. Fig. 5(a) is the generated pulse, sampled by a photoconductive gap $50 \mu \mathrm{m}$ away from the generating beam spot.
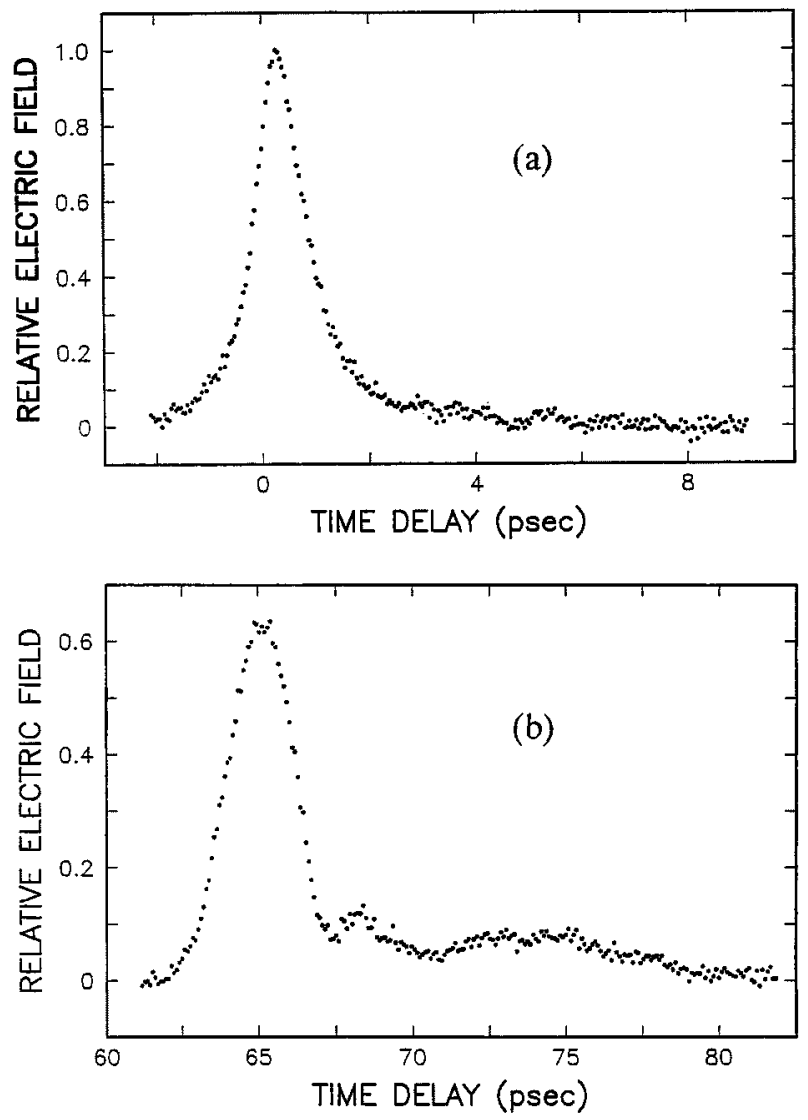

Fig. 5. (a) Initial electrical pulse. (b) Transmitted pulse.

After $8 \mathrm{~mm}$ of single-mode propagation, the pulse broadened and reshaped as shown in Fig. 5(b).

These data were used to determine the absorption and dispersion of the transmission line within the bandwidth of the electrical pulse. The waveforms in Fig. 5 were first numerically transformed into the frequency domain as shown in Fig. 6(a). In comparison to that of the input pulse, the attenuated spectrum of the transmitted pulse only extends to approximately $0.6 \mathrm{THz}$. Assuming a linear measurement system, the ratio of the measured output to input signal amplitude spectra $S_{0}(\omega) / S_{i}(\omega)$ is proportional to the ratio of the actual pulse spectra $E_{0}(\omega) / E_{i}(\omega)$. The amplitude component at frequency $\omega$ of the transmitted pulse $E_{0}(\omega)$ is determined by the amplitude absorption coefficient $\alpha$ and the wave vector $k$ as follows

$$
E_{0}(\omega)=E_{i}(\omega) \exp (i k z) \exp (-\alpha z)
$$

with $z$ indicating the propagation distance. The absorption coefficient $\alpha$ obtained from the spectra in Fig. 6(a) is shown and compared with theory in Fig. 6(b).

From lumped-parameter ac transmission line theory [18], $E_{0}(\omega)=E_{i}(\omega) \exp (i \gamma z)$, with

$$
\gamma=k+i \alpha=[(R+i \omega L)(i \omega C)]^{1 / 2}
$$

where $R=10 \Omega / \mathrm{mm}$, and where the inductance $L$ and capacitance $C$ per unit length are determined by the line impedance of $100 \Omega$ and the signal propagation velocity of $c / 2.45$. This result is shown in Fig. 6(b) and (c) as the dashed lines, where the high-frequency limit is only $10 \mathrm{GHz}$ and is in complete disagreement with the data out to the experimental limit of 0.6 

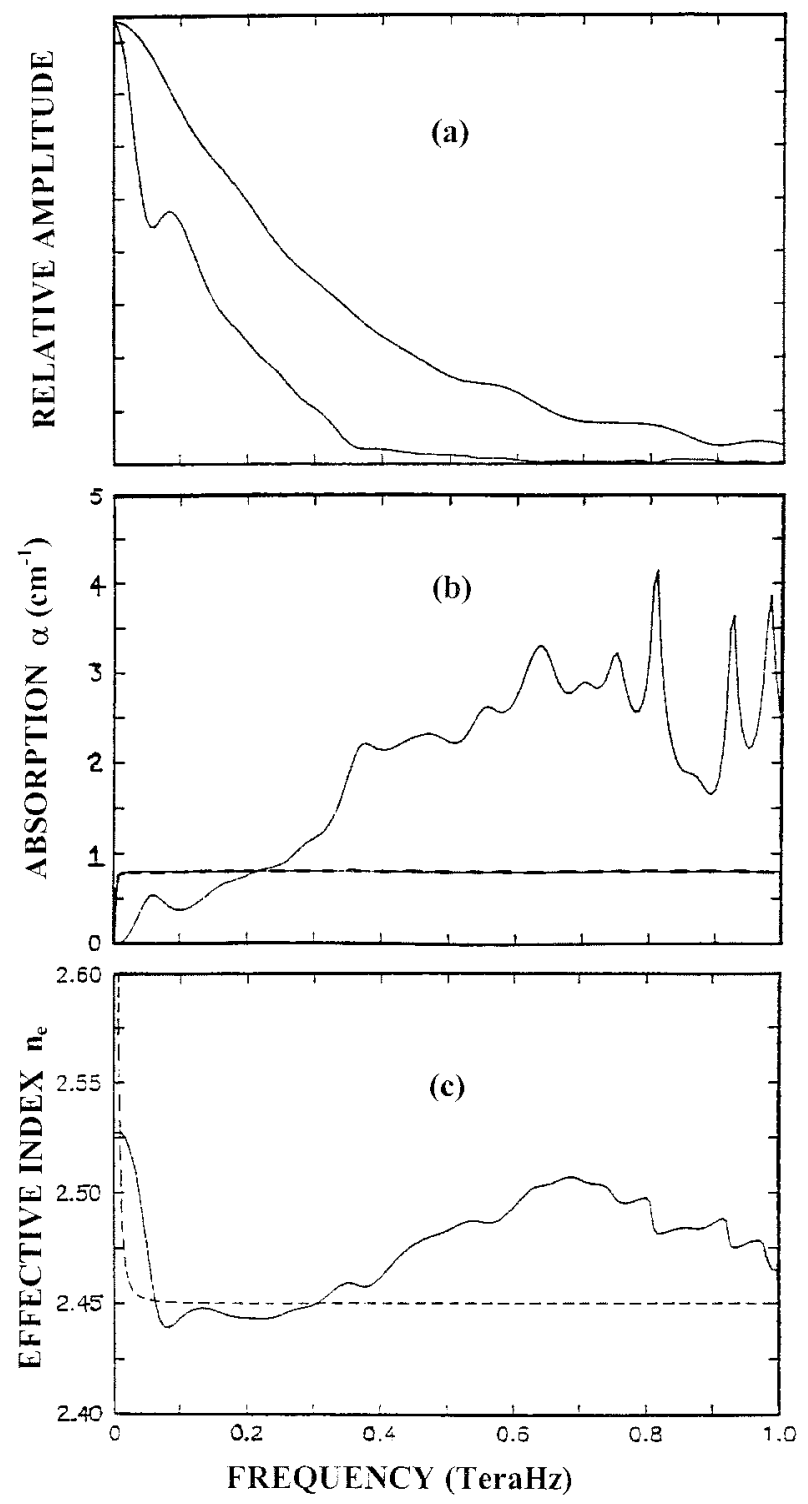

Fig. 6. (a) Amplitude spectrum of initial pulse (upper curve) and transmitted pulse (lower curve). (b) Measured absorption spectrum of transmission line; lower curve is calculated absorption. (c) Measured dispersion of transmission line (upper curve) compared to calculated dispersion.

THz. The measured absorption exhibits more structure than the theory, which appears as a step function. This could be do in part to the skin depth becoming less than the $0.5-\mu \mathrm{m}$ thickness of the aluminum line (at approximately $10 \mathrm{GHz}$ ), which in turn leads to a frequency-dependent $R(\omega)$. The effective index of refraction $n_{e}$ (dispersion) for the transmission line, obtained from the relationship $n_{e}=k c / \omega$, is shown in Fig. 6(c), along with the calculated effective index. The measured dispersion also differs from the theory in both the high- and low-frequency limits.

\section{Normal Superconducting Transmission Lines}

One of the early applications of THz-TDS was the study of superconducting niobium metal lines [19]. The optoelectronic technique works well in low-temperature cryostats, because the time resolution of the method is determined by the measurement of optical delay, and, therefore, requires only kilohertz response of the detection electronics. For the experiment, the same
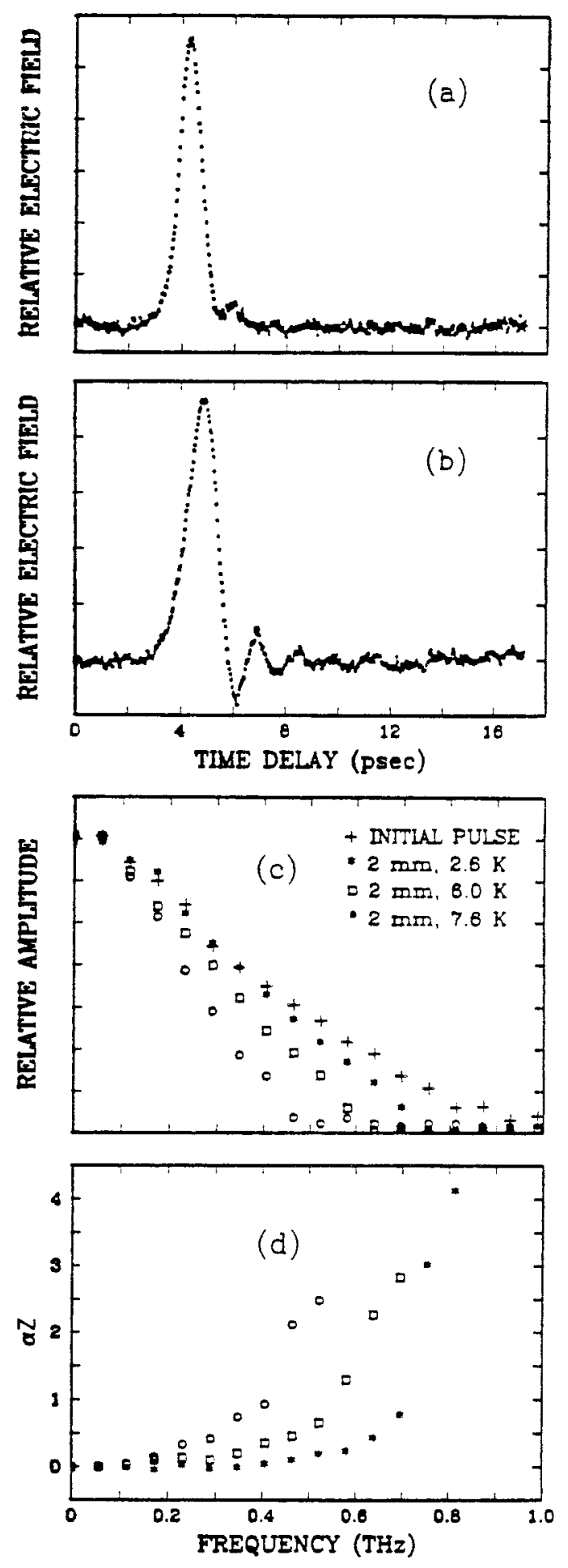

Fig. 7. (a) Initial electrical pulse. (b) Electrical pulse after $2 \mathrm{~mm}$ propagation on $\mathrm{Nb}$ superconducting transmission line at $2.6 \mathrm{~K}$. (c) Fourier transforms of initial and propagated pulses at different temperatures. (d) Absorption spectra of transmission line versus temperature.

pattern, as shown in Fig. 1(a), was fabricated in niobium metal on an ion-implanted SOS wafer.

Above the superconducting transition temperature $T_{c}=10 \mathrm{~K}$, the propagated pulse was severely broadened by the highly resistive $\mathrm{Nb}$ transmission line, compared to the input pulse shown in Fig. 7(a). At $2.6 \mathrm{~K}$, the propagated 
pulse shown in Fig. 7(b) developed a very characteristic ringing. When the input and propagated pulses are frequency analyzed, the resulting absorption coefficient has a sharp step at $0.7 \mathrm{THz}$, corresponding to the superconducting bandgap. Consequently, ordinary superconducting lines cannot be used to propagate 1-ps pulses.

\section{High $T_{c}$ Superconducting Lines}

As will be explained below, the Cherenkov radiation loss of coplanar transmission lines can be reduced to a manageable level by reducing the line dimensions to submicrometer levels. However, in this case the resistive losses of ordinary metal lines would significantly degrade the bandwidth. Ordinary superconductors can solve this problem for frequencies below their bandgaps of $0.7 \mathrm{THz}$ or less. However, for the high $T_{c}$ materials the band gap should be higher than $15 \mathrm{THz}$. Therefore, subps pulses could be transmitted down submicrometer high $T_{c}$ lines, if the dielectric substrates did not absorb at these frequencies.

An early study involving high $T_{c}$ materials was a pulse propagation study with an aluminum coplanar transmission line in proximity to an additional high $T_{c}$ ground plane, where loss-free propagation was observed [20]. Low-bandwidth (approximately $100 \mathrm{GHz}$ ) undistorted pulse propagation was reported on a $\mathrm{YBa}_{2} \mathrm{Cu}_{3} \mathrm{O}_{7-\delta}(\mathrm{YBCO})$ coplanar transmission line with $15-\mu \mathrm{m}$ linewidths and spacings, fabricated on a latticed-matched YSZ substrate [21], [42]. The low bandwidth was due to the wire bonding excitation scheme serving as a low-pass filter and the severe attenuation of the high-frequency components of the propagating pulse was due to the large absorption of the YSZ substrate. Because of the low effective bandwidth, very little temperature dependence was observed.

A later study presented 1-THz bandwidth results for a YBCO coplanar transmission line fabricated on lattice-matched lanthanum aluminate [22]. The two $20-\mu \mathrm{m}$-wide lines were separated by $10 \mu \mathrm{m}$. This study used a flip-chip geometry for excitation and electrooptic detection. It was observed that the losses on the YBCO line were lower than an equivalent gold line on the same substrate at temperatures below $50 \mathrm{~K}$.

A most recent 2-THz bandwidth study of $\mathrm{YBCO}$ and $\mathrm{Au} / \mathrm{YBCO}$ transmission lines on a lattice-matched $\mathrm{MgO}$ substrate found the disappointing result that at $77 \mathrm{~K}$ the attenuation and dispersion coefficients were larger for YBCO than for $\mathrm{Au}$ [23]. These results were obtained by photoconductive switching techniques using an amorphous photoconductive layer of GaAs, laser ablated directly onto the transmission line structure. The tentative explanation of this puzzling result, was that with respect to the two-fluid model, the surface losses involving the skin depth of YBCO scaled as the frequency squared, whereas for a normal metal it scales as the square root of the frequency.

At this time, YBCO transmission lines have not demonstrated their superiority to normal metal lines for bandwidths significantly above $1 \mathrm{THz}$. This situation must be resolved, if high $T_{c}$ lines are going to realize their promise as the future material for the VLSI interconnect with dimensions below $1 \mu \mathrm{m}$ and $\mathrm{THz}$ bandwidths.

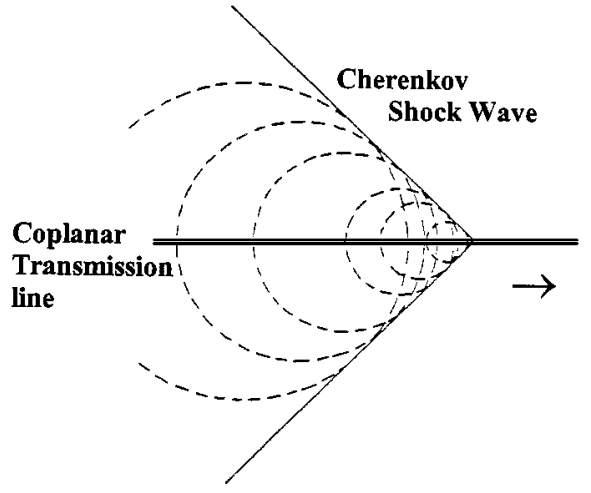

Fig. 8. Top view of the Cherenkov shock wave cone in the dielectric half-space laying in the plane of the figure. The dipolar pulse located at the apex of the Cherenkov cone is propagating at $\mathrm{c} / 2.45$ down the coplanar transmission line on the surface of the sapphire substrate. The dashed circles represent the near-field radiation into the sapphire from the moving dipole. This radiation is propagating at the phase velocity $\mathrm{c} / 3.3$ of sapphire.

\section{CHERENKOV SHOCK WAVE RADIATION FROM TRANSMISSION LINES}

By studying the frequency-dependent loss on an Al transmission line on SOS, where the coplanar lines were separated by $15 \mu \mathrm{m}$, a powerful radiation process due to a type of Cherenkov radiation was observed [24]. The initial analysis of Cherenkov radiation was for electric monopoles, but the physical picture holds true for higher order moments as well [25]. Here, the considered electrical pulses were produced by transferring approximately 2000 electrons between the two lines at the generation site. Consequently, two dipoles with charges of $1000 e$ and separations of $15 \mu \mathrm{m}$ move down opposite directions of the line at the measured group velocity of $c / 2.45$. This velocity is significantly faster than the phase velocity of approximately $c / 3.3$ for $\mathrm{THz}$ radiation in sapphire. Thus, in the dielectric the situation shown in Fig. 8 generates radiation of an electromagnetic shock wave in the form of a Cherenkov-type cone.

THz-TDS measurements have characterized this loss process, which can be so severe that after propagating only $1 \mathrm{~mm}$, power at $0.8 \mathrm{THz}$ is reduced to $1 / e$ of its original value [24]. Previous microwave studies have calculated the radiative loss as the leaky waveguide effect for single-frequency transmission on coplanar metal lines on dielectrics [26]; these results were confirmed by experimental measurements up to $1 \mathrm{GHz}$, where the radiation loss is only $10^{-9}$ of that reported here. The observed frequencydependent absorption [24] remains in good agreement with this calculation, even though the measurement extends up to $1 \mathrm{THz}$.

These time-domain experiments add another dimension to this process in that all of the Cherenkov radiation over the bandwidth of the propagating pulse is phase coherent resulting in the radiation of the conical shock wave illustrated schematically in Fig. 8. The short electromagnetic pulse associated with this shock wave has been directly observed [27]. Using conical optics, this shock wave has been coupled out of the dielectric substrate and demonstrated as a source of freely propagating $\mathrm{THz}$ radiation [28].

Fig. 9(a) shows the measured initial subps electrical pulse for which the spatial separation between the exciting and sampling 

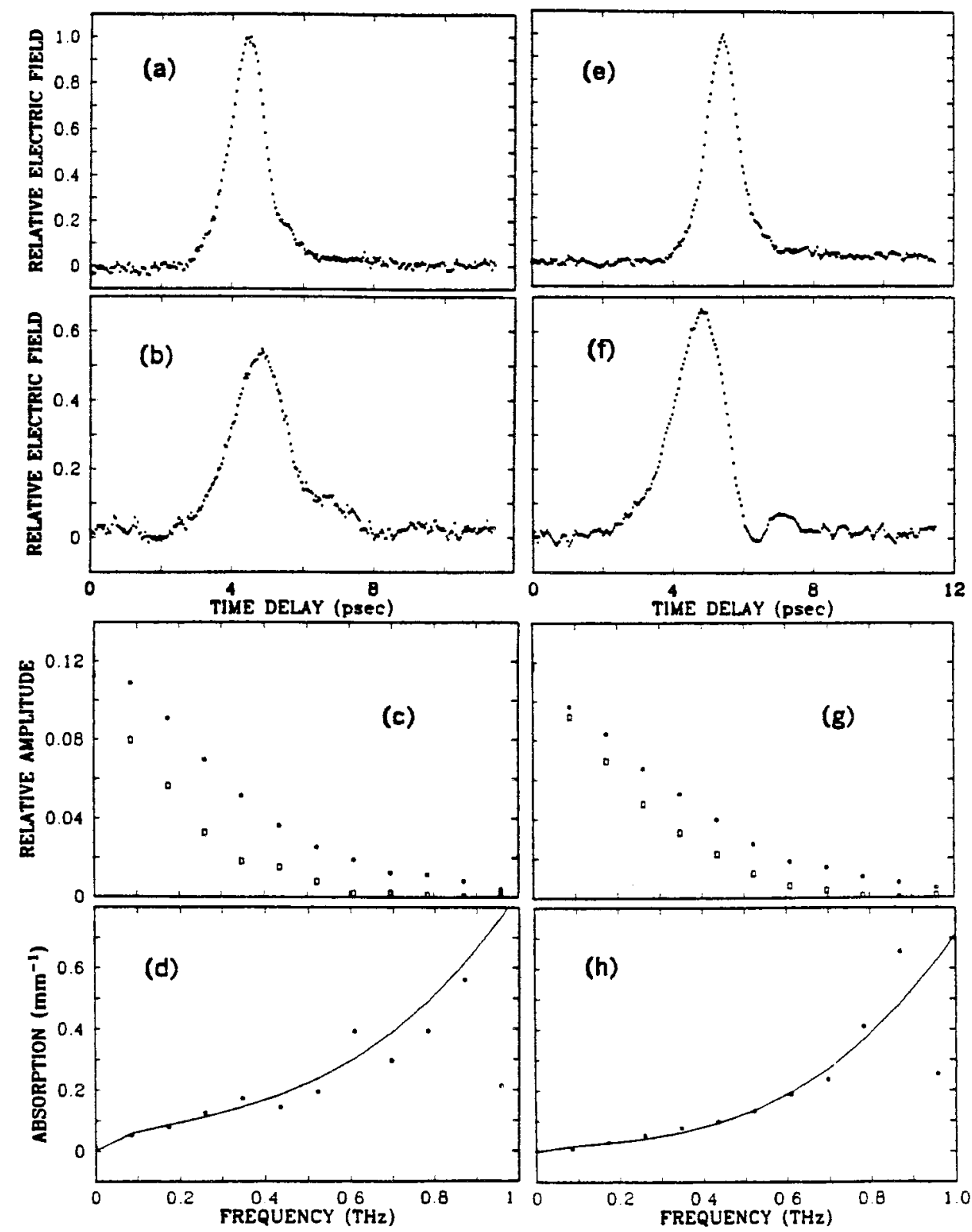

Fig. 9. (a) Room temperature, measured input pulse. (b) Measured pulse after $6 \mathrm{~mm}$ propagation. (c) Amplitude spectrum of input pulse (dots, upper trace) compared to propagated pulse (squares). (d) Experimental amplitude absorption coefficient (dots) compared with theory. (e) $2.5 \mathrm{~K}$ measured input pulse. (f) 2.5 $\mathrm{K}$ pulse after $6 \mathrm{~mm}$ propagation. (g) Amplitude spectrum of $2.5 \mathrm{~K}$ input pulse (dots, upper trace) compared to propagated pulse (squares). (h) Experimental $2.5 \mathrm{~K}$ amplitude absorption coefficient (dots) compared with theory.

beams was approximately $50 \mu \mathrm{m}$. When the sliding contact was moved $6 \mathrm{~mm}$ from the optical sampling gap, the pulse propagation effects shown in Fig. 9(b) were observed. The Fourier analyzes of these pulses are shown in Fig. 9(c), where it is seen that the amplitude spectrum of the initial pulse extends beyond $1 \mathrm{THz}$ and that the transmitted pulse has suffered a significant frequency-dependent loss. From these spectra, the absorption coefficient is obtained as a function of frequency, as shown in Fig. 9(d). This large absorption needs an explanation. The absorption of the thin $0.5-\mu \mathrm{m}$ layer of silicon is relatively insignificant [7]. The same is true for the sapphire substrate, whose absorption coefficient in this frequency range is also insignificant [7]. Therefore, the initial explanation was that this absorption was due to the aluminum metal film.
In order to test this assumption, the resistivity of the $\mathrm{Al}$ lines was reduced by approximately 12 times by cooling the transmission line to liquid helium temperatures. Instead of the expected reduction in the absorption coefficient by at least $1 /(12)^{1 / 2}$, due to the consequent reduction in the surface resistance controlled by the frequency-dependent skin effect, the results shown in Fig. 9(e)-(h) were obtained. The initial pulse looks almost identical to the room-temperature result. The propagated pulse illustrated in Fig. 9(f) broadened considerably and developed a slight ringing. In addition, as shown in Fig. 9(g), the Fourier spectra of the input and propagated pulses at $2.5 \mathrm{~K}$ look very similar to the room-temperature results. This comparison is made more precise when the absorption coefficient is plotted [Fig. 9(h)]. Thus, it is clear that the main component of the observed loss is not 
due to the aluminum metal film. Evaluating the predicted [26] loss for these conditions, the amplitude absorption coefficient is found to be $\alpha=\left(0.4 \mathrm{~mm}^{-1}\right) f^{3}$, where $f$ is the frequency in THz. This result is to be compared with the measurements in Fig. 9(d) and (h). The solid line fit to the data in Fig. 9(d) is given by the relationship $\alpha=\left(0.2 \mathrm{~mm}^{-1}\right) f^{1 / 2}+\left(0.65 \mathrm{~mm}^{-1}\right) f^{3}$. The first term gives the frequency-dependent loss due to the resistive skin effect for the $\mathrm{Al}$ lines, while the second term with the $f^{3}$ dependence describes the loss due to the radiation. In Fig. 9(h), the solid line fit to the measured absorption is again the sum of two terms, $\alpha=\left(0.06 \mathrm{~mm}^{-1}\right) f^{1 / 2}+\left(0.65 \mathrm{~mm}^{-1}\right) f^{3}$. It is to be noted that the radiative term, as expected, remained the same, while the resistive loss term decreased by approximately $1 /(12)^{1 / 2}$, corresponding to the reduction of 12 in the resistance of the lines. The measured loss due to the radiation is approximately 1.5 times the calculated value; this good agreement and especially the cubic frequency dependence confirms the nature of the effect responsible for the excess absorption.

Other groups have studied the loss mechanisms involved in the propagation of ultrashort electrical pulses on coplanar transmission lines [29]-[31]. It is generally agreed that the main absorption loss at lower frequencies $(<200 \mathrm{GHz})$ is through conduction losses in the lines ( $\propto f^{1 / 2}$, where $f$ is the frequency), whereas the high-frequency absorption $\left(\propto f^{3}\right)$ is predominantly due to Cherenkov radiation. Later work has shown that in the full-wave analysis approach for the absorption, the effective dielectric constant is slightly frequency dependent, thereby modifying the absorption from a strict $f^{3}$ dependence [30].

\section{A. Methods to Eliminate Cherenkov Radiation from Transmission Lines}

The Cherenkov radiation loss was eliminated by the use of silicon-on-insulator materials with a microstrip geometry [32] and thin-film microstrip lines [33], but the total observed loss owing to the dielectric and the metal was still approximately the same as that for the coplanar transmission line. A variety of transmission line structures have been designed to reduce the permittivity mismatch responsible for the Cherenkov radiation [34]-[37]. The ideal transmission line would have no substrate/superstrate mismatch. Along these lines, coplanar air transmission (CAT) lines shown in Fig. 10 were fabricated [34]; these coplanar striplines are edge supported by a 200-nm layer of $\mathrm{SiO}_{2}$ on $\mathrm{GaAs}$. The GaAs below the transmission line is etched away leaving the striplines supported in free space. Rise times as short as 0.8 ps after a propagation length of $2.8 \mathrm{~mm}$ were obtained [34]. Coplanar striplines have also been fabricated on low-permittivity substrates and membranes, on the order of micrometers thick, demonstrating significantly reduced loss and higher bandwidths [35]-[37], e.g., Figs. 11 and 12 .

A new approach to the reduction of radiative loss in ultrafast coplanar transmission lines has been demonstrated [38]. Instead of reducing the permittivity mismatch, the mode characteristics of a three-line stripline were utilized to generate a quadrupole ultrashort electrical pulse with significantly reduced absorption and dispersion compared to that of the dipole pulse. As shown in Fig. 13(a), through appropriate bias of the striplines, either the

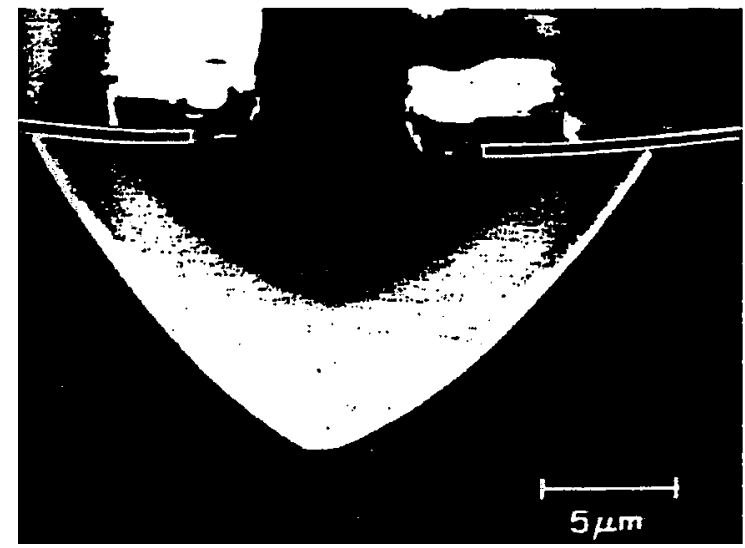

Fig. 10. "Scanning electron micrograph of the etched CAT line. Linewidth and spacing is $5 \mu \mathrm{m}$. The front edge of the $\mathrm{SiO}_{2}$ layer has been highlighted for clarity." From [34].

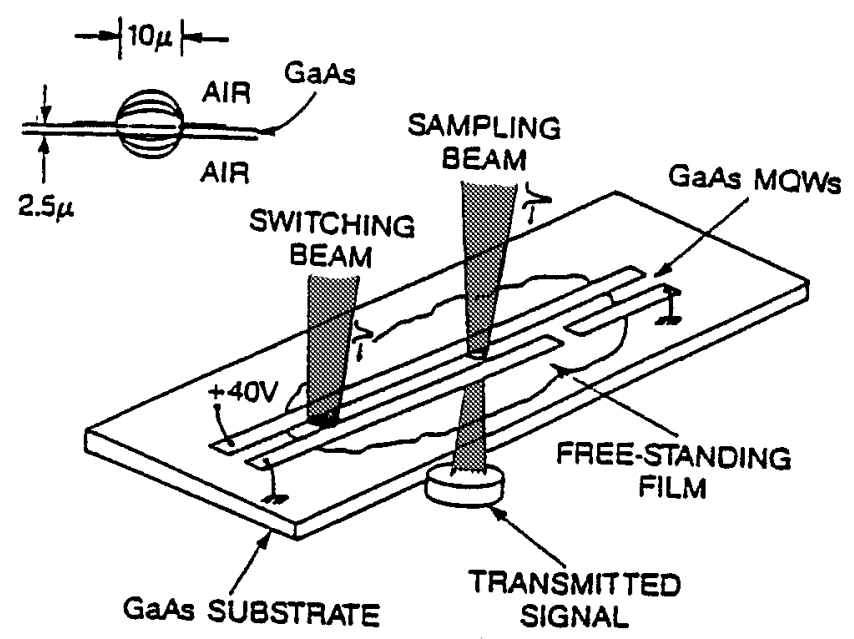

Fig. 11. "Sample structure. A $10 \mu \mathrm{m}$ gold coplanar stripline which is $5 \mathrm{~mm}$ long is patterned onto a sample composed of $0.7 \mu \mathrm{m}$ of GaAs quantum well on top of $1.97 \mu \mathrm{m}$ of stop etch AlGaAs layer. The substrate is removed in a $1 \times 1.3$ $\mathrm{mm}$ area. A pump beam excites the stripline and the probe beam samples the excitonic electroabsorption response anywhere in the circuit. The inset indicates that the field lines propagate in air on both sides of the dielectric." From [35].

even mode, where the electric fields between each gap are parallel with a dipole field pattern, or the odd mode, which has the electric fields in the gaps anti-parallel with a quadrupole field pattern, can be exclusively excited. A series of ultrashort electrical pulse data for both the dipole and quadrupole configurations is shown in Fig. 13(b), with pulse propagation distances from 0.2 to $5.2 \mathrm{~mm}$.

Via THz-TDS analysis, the obtained amplitude absorption coefficients $\alpha(f)$, for the quadrupole pulse and dipole pulse are shown in Fig. 14(a). The absorption for the quadrupole is dramatically reduced over that of the dipole; at $0.8 \mathrm{THz}$ a factor of two difference between the two coefficients is observed. Both curves are fit with the relationship $\alpha(f)=A_{\text {res }} f^{1 / 2}+A_{\text {rad }} f^{3}$, where the resistive $A_{\text {res }}$ and the radiative $A_{\text {rad }}$ loss coefficients are adjustable parameters. This relation fits both curves reasonably well, with the two resistive coefficients having values that agree to within about $15 \%$. For the dipole pulse, $A_{\text {res }}$ is $6 \mathrm{~cm}^{-1}$, compared to $7 \mathrm{~cm}^{-1}$ for the quadrupole. For the dipole pulse, 


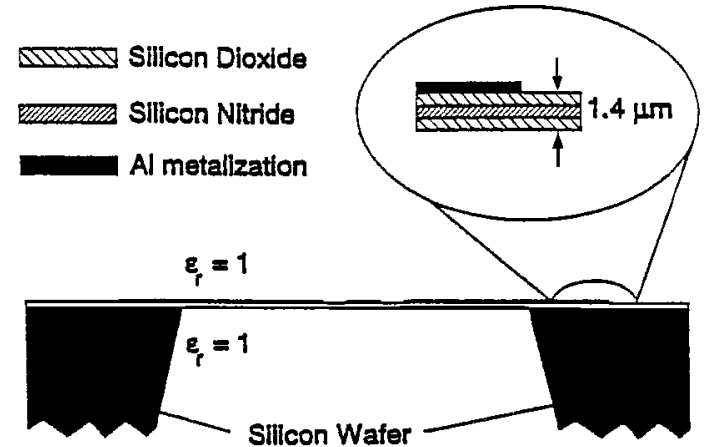

Fig. 12. "Cross-sectional view of ultra-low-distortion coplanar waveguide with a $1.4-\mu \mathrm{m}$ thick substrate of silicon dioxide and silicon nitride. Waveguide had a center strip width of $40 \mu \mathrm{m}$ and spacings of $25 \mu \mathrm{m}$." From [37].
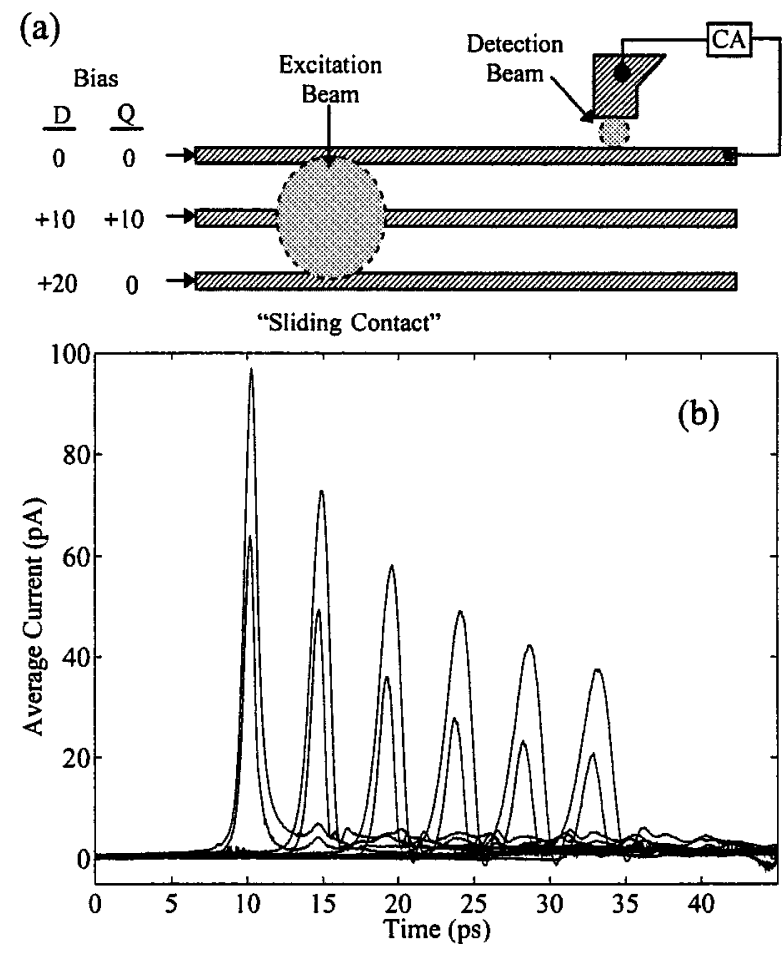

Fig. 13. (a) Three-line coplanar transmission line with sliding contact excitation and side gap detection. The aluminum strip lines are $4 \mu \mathrm{m}$ wide separated by $15 \mu \mathrm{m}$. Quadrupole electrical pulses are generated with bias $\mathrm{Q}$, dipole pulses with bias D. (b) Measured quadrupole (lower curves) and dipole electrical pulses at propagation lengths of $0.2,1.2,2.2,3.2,4.2$, and $5.2 \mathrm{~mm}$.

the strong $f^{3}$ dependence is seen with a radiative coefficient $A_{\text {rad }}=32 \mathrm{~cm}^{-1}$, whereas the quadrupole pulse has the much smaller coefficient $A_{\mathrm{rad}}=3 \mathrm{~cm}^{-1}$. This order of magnitude reduction clearly demonstrates that the radiative loss for an electric quadrupole pulse is significantly less than that for a dipole pulse. The index of refraction for the two modes of the transmission line is plotted for the same set of pulses in Fig. 14(b). The frequency-dependent part of the index for the quadrupole pulse is less than one-third that for the dipole pulse.

It is instructive to compare the measured $A_{\text {rad }}=32 \mathrm{~cm}^{-1}$ for the 30- $\mu \mathrm{m}$-sized dipole with that expected from the measurements of Fig. 9, where $A_{\text {rad }}=6.5 \mathrm{~cm}^{-1}$ for a $15-\mu \mathrm{m}$ dipole. Because the radiation loss is proportional to the square
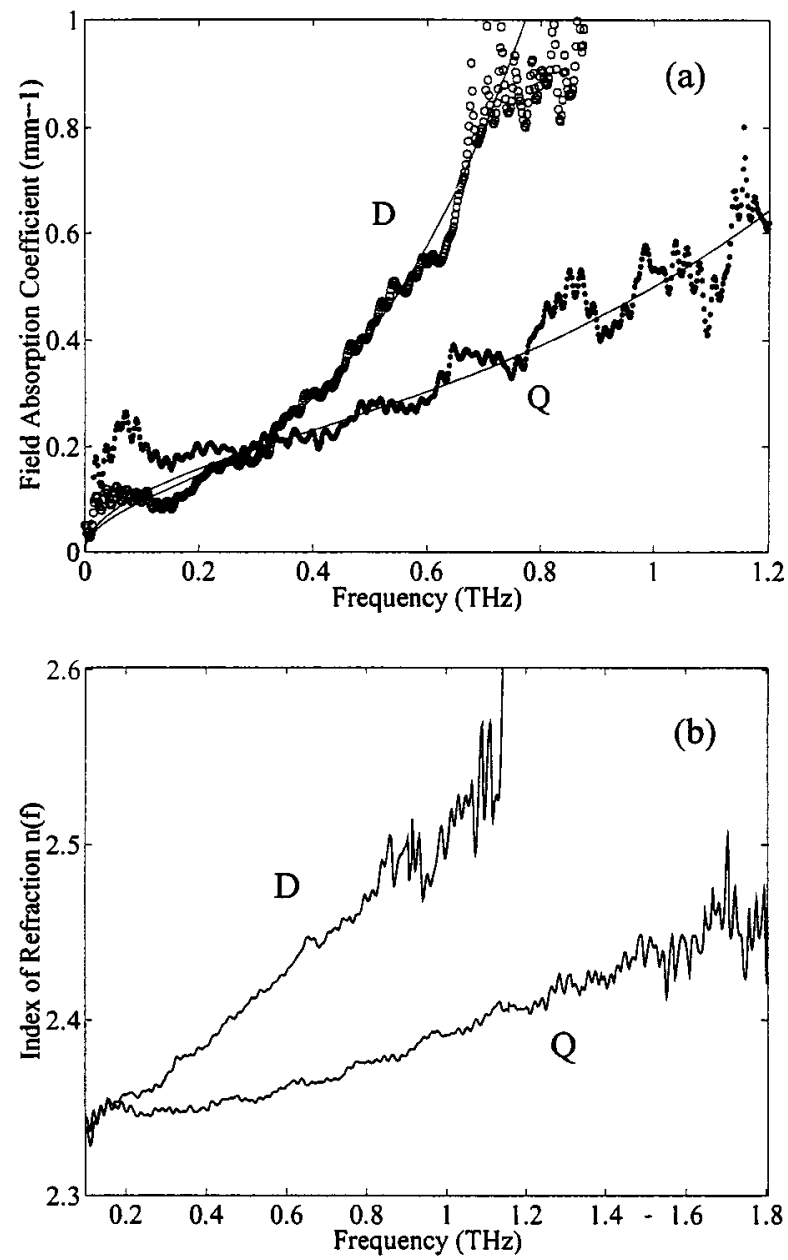

Fig. 14. (a) Measured amplitude absorption coefficient overlaid with the fit to the data for $(\mathrm{Q})$ quadrupole (odd mode) and (D) dipole (even mode) pulses. (b) Measured index of refraction for the (Q) quadrupole and (D) dipole pulses.

of this length, Fig. 9 would predict an $A_{\text {rad }}=28 \mathrm{~cm}^{-1}$ for a $30-\mu \mathrm{m}$-sized dipole in good agreement with observation.

\section{THz-TDS CHARACTERIZATION OF WAVEGUIDES}

The technical capability to optoelectronically transmit and detect single-cycle pulses of freely propagating $\mathrm{THz}$ electromagnetic radiation has generated much interest in the guided-wave propagation of such pulses. At the present time, the highest guided-wave performance has been obtained with a $240-\mu \mathrm{m}$-diameter stainless-steel waveguide over the frequency range from 0.8 to $3.5 \mathrm{THz}$ and with a power absorption coefficient of less than $1 \mathrm{~cm}^{-1}$ [11]. This is more than an order of magnitude less absorption than for the transmission lines discussed above.

\section{A. Metal Waveguides}

Experimental investigations using quasi-optical methods to efficiently couple freely propagating subps pulses of $\mathrm{THz}$ radiation into submillimeter circular metal tubes used as waveguides and to consequently measure the transmitted pulses from these waveguides have been recently reported [11], [12]. Within the passband of the waveguide, the measured power coupling into 


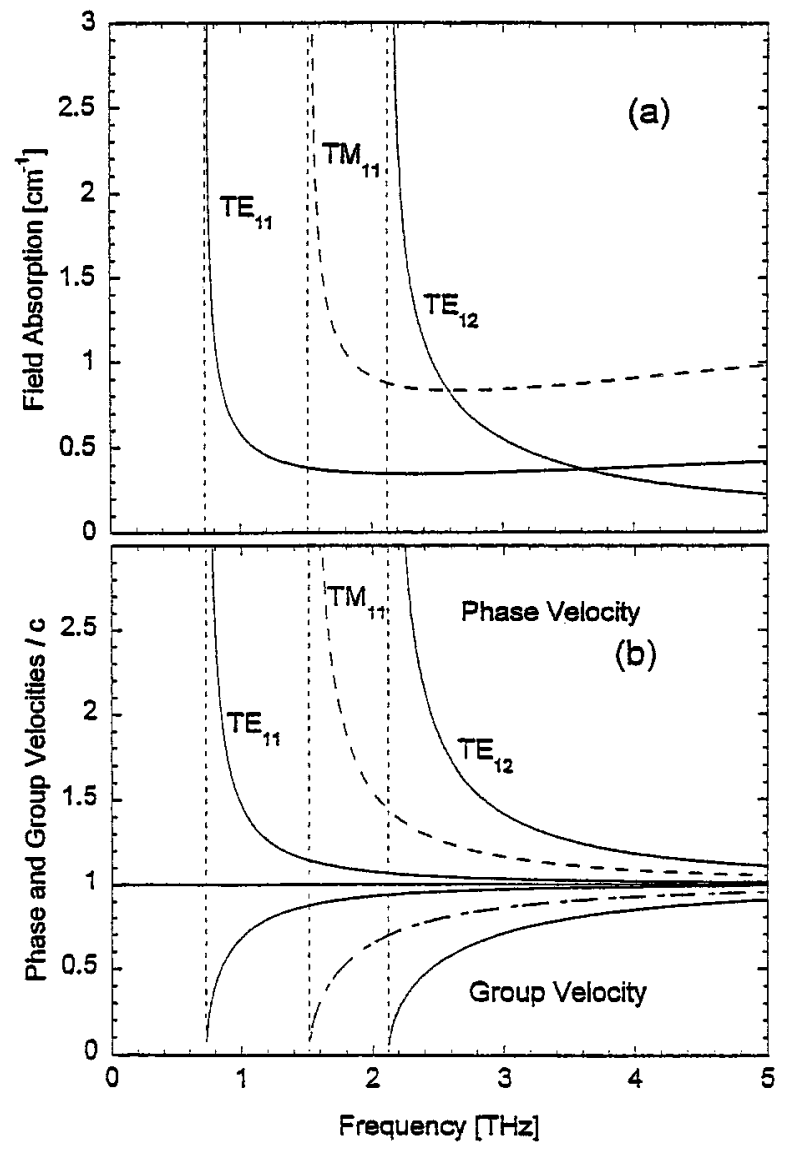

Fig. 15. (a) Field absorption and (b) phase and group velocities for the coupled three modes in a $240-\mu \mathrm{m}$-diameter stainless-steel waveguide.

the waveguide was typically $40 \%$ of the incoming $\mathrm{THz}$ power. Dispersive propagation with low loss over the frequency band from 0.65 to $3.5 \mathrm{THz}$ was observed with frequency-dependent group velocities $v_{g}$ ranging from $c / 4$ to $c$ and phase velocities $v_{p}$ from $4 c$ to $c$, where $v_{g} v_{p}=c^{2}$. Even though the input spectrum overlapped the cutoff frequencies of more than 25 waveguide modes, the linearly polarized incoming $\mathrm{THz}$ pulses only significantly coupled into the $\mathrm{TE}_{11}, \mathrm{TM}_{11}$, and $\mathrm{TE}_{12}$ modes. The observations are in agreement with waveguide theory and were obtained using 24-mm-long and 4-mm-long stainless-steel tubes with inside diameters of $240 \mathrm{~m}$ and $280 \mu \mathrm{m}$, respectively. As shown in Fig. 15 [18], [39], [40], at $1 \mathrm{THz}$ the power absorption coefficient for the dominant $\mathrm{TE}_{11}$ mode is $\alpha=0.7 \mathrm{~cm}^{-1}$ for a $280-\mu \mathrm{m}$-diameter SS waveguide.

The ultrafast optoelectronic measurements were performed by THz-TDS with a simple modification of the THz optoelectronic beam system [10] shown in Fig. 16(a), consisting of an optoelectronic transmitter and receiver along with $\mathrm{THz}$ beam shaping and steering optics. For the waveguide experiment, a lens-waveguide-lens system was placed at the $\mathrm{THz}$ beam waist between the two parabolic reflectors. For this system, the hyperhemispherical silicon lenses [7], [10] focus the $\mathrm{THz}$ beam to a frequency-independent $1 / e$ waist diameter of $200 \mu \mathrm{m}$. The focused $\mathrm{THz}$ beam is coupled into and propagated through the circular metal waveguide and coupled out with the second silicon lens.
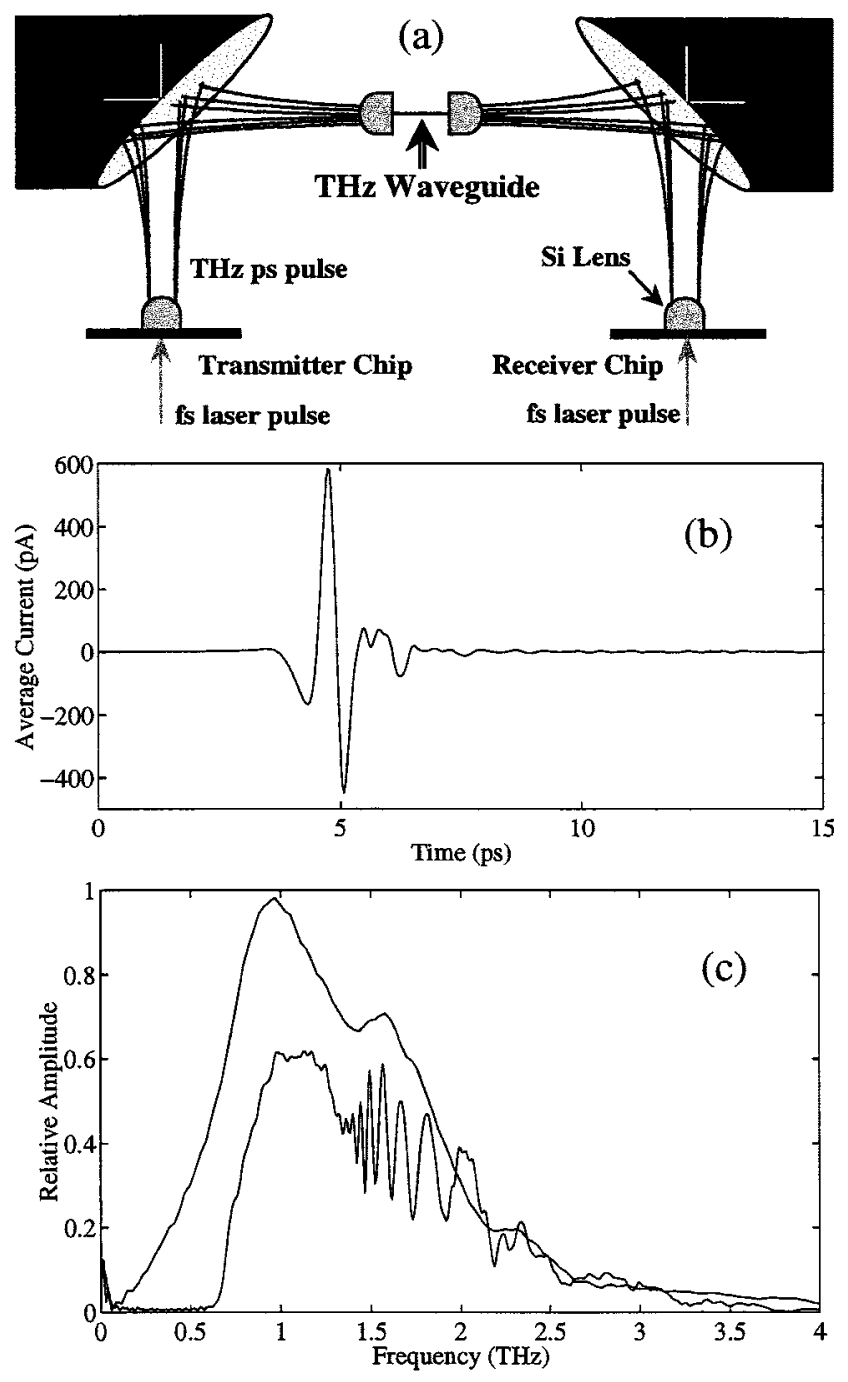

Fig. 16. (a) Schematic diagram of the optoelectronic THz-TDS system incorporating quasi-optical coupling to the investigated $\mathrm{THz}$ waveguide. (b) Measured reference $\mathrm{THz}$ pulse. (c) Relative amplitude spectrum of the reference pulse compared to that of the transmitted pulse.

The reference $\mathrm{THz}$ pulse shown in Fig. 16(b) is obtained by removing the waveguide and moving the two silicon lenses to their confocal position. Fig. 16(c) shows that the useful amplitude spectrum extends from 0.1 to $4 \mathrm{THz}$. The observed transmitted signal from the 24-mm-long waveguide dramatically demonstrated the strong group velocity dispersion of the waveguide [11], [12]. The 1-ps input pulse was stretched to about $70 \mathrm{ps}$, with the high frequencies arriving earlier in time corresponding to negative chirp.

Compared to the 24-mm-long waveguide, the transmitted pulse from a 4-mm-long waveguide displays much less cumulative dispersion in Fig. 17(a) (dots); the pulse is only spread over $30 \mathrm{ps}$ and shows fewer oscillations. The spectrum of this pulse, Fig. 17(b) (dots), shows the lowest frequency cutoff at about $0.65 \mathrm{THz}$, which is also calculated (for SS) to be $0.65 \mathrm{THz}$ for the $\mathrm{TE}_{11}$ mode. The comparison of the relative amplitude spectrum of this pulse with that of the reference pulse, where they both have the same normalization, illustrates the excellent amplitude coupling of the freely propagating $\mathrm{THz}$ pulse into 

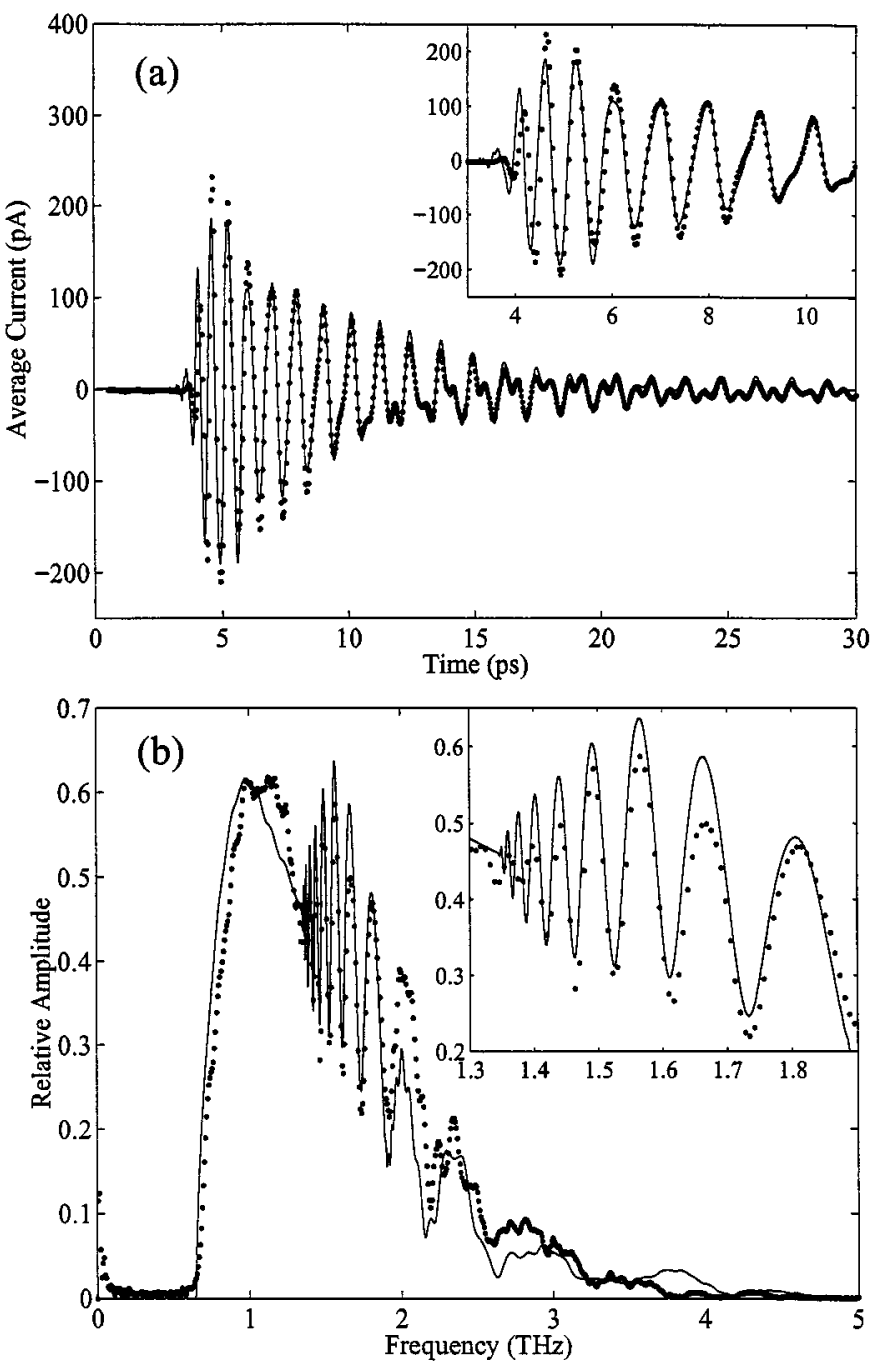

Fig. 17. (a) Measured $\mathrm{THz}$ pulse (dots) transmitted through a 4-mm-long, $280-\mu$ m-diameter stainless-steel waveguide. The inset shows the $\mathrm{THz}$ pulse on an expanded time scale. (b) Amplitude spectrum (dots) of the measured transmitted pulse [Fig. 17(a)]. The inset shows the amplitude spectrum on an expanded frequency scale. The solid curves are the theoretical predictions.

the waveguide. Multimode interference is observed to be stronger in the shorter waveguide, starting at about $1.3 \mathrm{THz}$. The dominant $\mathrm{TE}_{11}$ mode has a cutoff frequency of $0.65 \mathrm{THz}$ (77\%), the second coupled mode is the $\mathrm{TM}_{11}$ with a cutoff at $1.31 \mathrm{THz}(20 \%)$, and the third weakly coupled mode is the $\mathrm{TE}_{12}$ with a cutoff at $1.81 \mathrm{THz}(3 \%)$, where the percentage of the total coupled power into each mode is in parentheses.

Using the spectrum of the reference pulse, a theoretical calculation for the output spectrum from the 4-mm waveguide was performed which included the three coupled modes, their respective cutoff frequencies, as well as their frequency-dependent complex propagation vectors, obtainable from Fig. 15. This calculation overlays as the solid line the measured spectra in Fig. 17(b), where good agreement is obtained for the interference oscillations as well as the other features of the spectra. The calculated time-domain output pulse (solid line) is given by the inverse Fourier transform of the calculated complex output amplitude spectrum $E_{\text {out }}(\omega, z)$ and is compared favorably with experiment in Fig. 17(a).
The observed extreme broadening of ps $\mathrm{THz}$ pulses due to their waveguide propagation is caused by the extreme group velocity dispersion (shown in Fig. 15) near the cutoff frequencies. This situation does not occur for the TEM mode of a two-wire transmission line or for the TEM mode of a coaxial waveguide, which do not have a cutoff frequency. Such TEM transmission has the group and phase velocities determined solely by the surrounding dielectric. However, quasi-optical coupling techniques would not be effective for such modes, because of the small overlap integral.

\section{B. Dielectric Waveguides}

An alternative approach to low-loss, guided-wave, $\mathrm{THz}$ propagation would be to use dielectric waveguides. Such waveguides do not have the sharp low-frequency cutoff of metal waveguides and would thereby extend the low-frequency limit. Given a suitable low-loss dielectric, such as high-resistivity silicon with a power absorption coefficient of much less than $0.05 \mathrm{~cm}^{-1}$ up to $2 \mathrm{THz}$ [7], dielectric waveguides could have much less absorption than metal waveguides. In addition, due to boundary considerations, it should be possible to essentially completely couple linearly polarized $\mathrm{THz}$ radiation into the single dominant $\mathrm{HE}_{11}$ mode for dielectric waveguides. Such dielectric single-mode $\mathrm{THz}$ waveguides would have the promise of an extremely low-loss, flexible interconnect and communications channel, with advantages similar to those of single-mode optical fiber.

Demonstrations of single-mode propagation of subps $\mathrm{THz}$ pulses in dielectric waveguides (fibers) have been recently reported [13]. The observed dominance of the $\mathrm{HE}_{11}$ mode, despite the fiber diameters allowing for multimode propagation, is attributable to the efficient, quasi-optical, free space to waveguide coupling [41]. These demonstrations show the viability of the single-mode $\mathrm{THz}$ fiber interconnect. The fact that the diameters $(325,250$, and $150 \mu \mathrm{m})$ of the $\mathrm{THz}$ fibers used are similar to those of optical fibers, including the core and cladding, gives the $\mathrm{THz}$ fibers similar flexibility and handling properties.

The experimental arrangement employed for coupling of free-space single-cycle pulses of $\mathrm{THz}$ radiation into the fiber was similar to that shown in Fig. 16(a). The measured $\mathrm{THz}$ pulse transmitted through the $325-\mu \mathrm{m}$ sapphire fiber is presented in Fig. 18(a). For all fibers, the incident 0.6-ps $\mathrm{THz}$ pulse undergoes considerable absorptive and dispersive reshaping and broadening due to the waveguide propagation, and emerges strongly frequency chirped. The degree of chirping is dependent on the fiber diameter, with the $325-\mu \mathrm{m}$ fiber stretching the pulse to $\approx 10 \mathrm{ps,} \mathrm{while} \mathrm{the} \mathrm{smallest} \mathrm{fiber,}$ $150 \mu \mathrm{m}$, leads to a pulse duration of greater than $30 \mathrm{ps}$. The decreased signal obtained for the smaller fibers is attributed to the lower coupling efficiency. The amplitude spectrum obtained for transmission through the $325-\mu \mathrm{m}$ fiber is shown in Fig. 18(b), together with the corresponding reference spectrum; the considerable attenuation of the higher frequencies is due to the absorption of sapphire.

The reference pulse was subjected to a theoretical propagation through the $325-\mu \mathrm{m}$ fiber, resulting in the calculated waveform shown in Fig. 18(a), and the calculated transmission spectrum shown in Fig. 18(b). The pulse propagation was obtained 

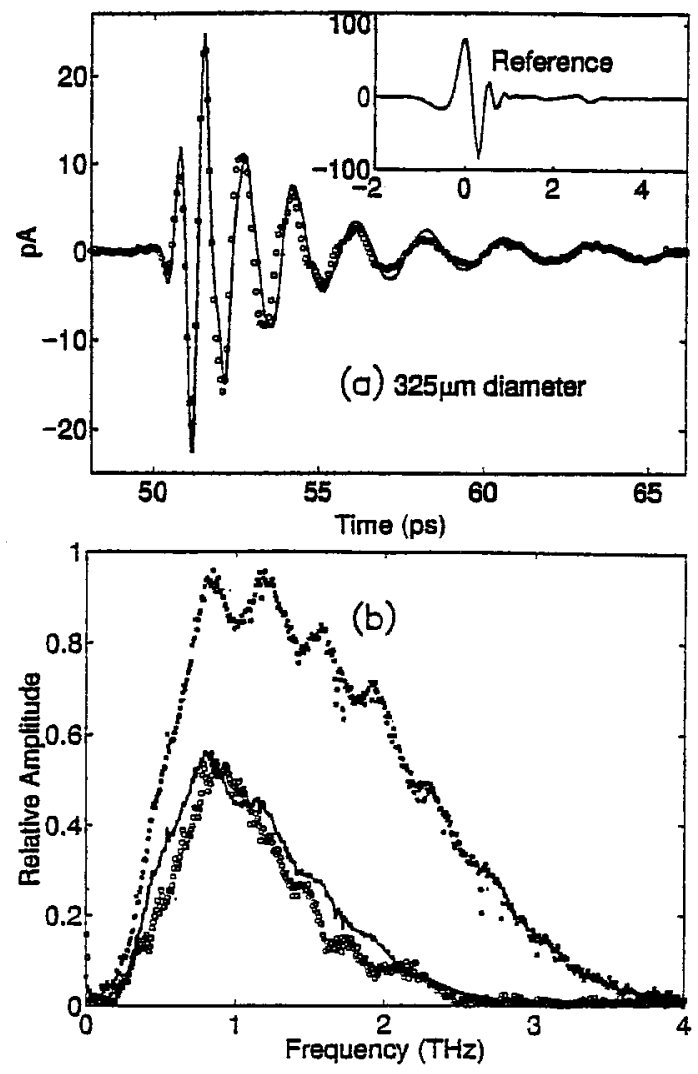

Fig. 18. (a) The THz pulse transmitted through a $325-\mu$ m-diameter sapphire fiber of length $7.3 \mathrm{~mm}$. The solid line is the calculated signal, while the circles are the measured transmitted pulse. The measured reference input pulse is shown in the inset. (b) Transmitted amplitude spectra. The solid line is the calculated spectrum, while the empty and solid circles (upper curve) are the measured transmission and reference input spectra, respectively.

by a frequency-domain analysis in terms of the single $\mathrm{HE}_{11}$ waveguide mode as follows:

$$
E_{\text {out }}(\omega)=E_{\text {ref }}(\omega) T C_{11}^{2} \exp \left\{i\left(k_{11}-k_{0}\right) z\right\} \exp \left\{-\alpha_{11} z / 2\right\}
$$

where the propagation constant of the fiber is $k_{11}$ and the power absorption coefficient is $\alpha_{11}$. As shown in Fig. 19(a), above 0.5 $\mathrm{THz}, \alpha_{11}$ closely follows that of the ordinary ray in bulk sapphire [7]. However, below 0.2 and $0.4 \mathrm{THz}, \alpha_{11}$ is significantly reduced, because for these lower frequencies a significant fraction of the power propagates outside the absorbing fiber. Although the small frequency-dependent variation of the sapphire refractive index was included in the calculations, it was found that the pulse dispersion was almost entirely due to the waveguide propagation characteristics. The group and phase velocities for the 325- and 150- $\mu \mathrm{m}$ fibers are shown in Fig. 19(b), from which it can be clearly observed that the phase velocity approaches that of bulk sapphire at the higher frequencies $(>2$ $\mathrm{THz}$ ), while for the very low frequency end of the spectrum the velocity approaches that of free space. The transmission coefficient $T \approx 0.74$ accounts for reflection losses at the fiber input and output, while $C_{11}$ is the overlap integral for the $\mathrm{HE}_{11}$ mode. The calculated $C_{11}$ are shown in Fig. 19(a) for both the 325and $150-\mu \mathrm{m}$ fibers. The second factor of $C_{11}$ in (8) accounts for the coupling out of the fiber and onto the detector, for the symmetric and confocal experimental arrangement. As can be seen

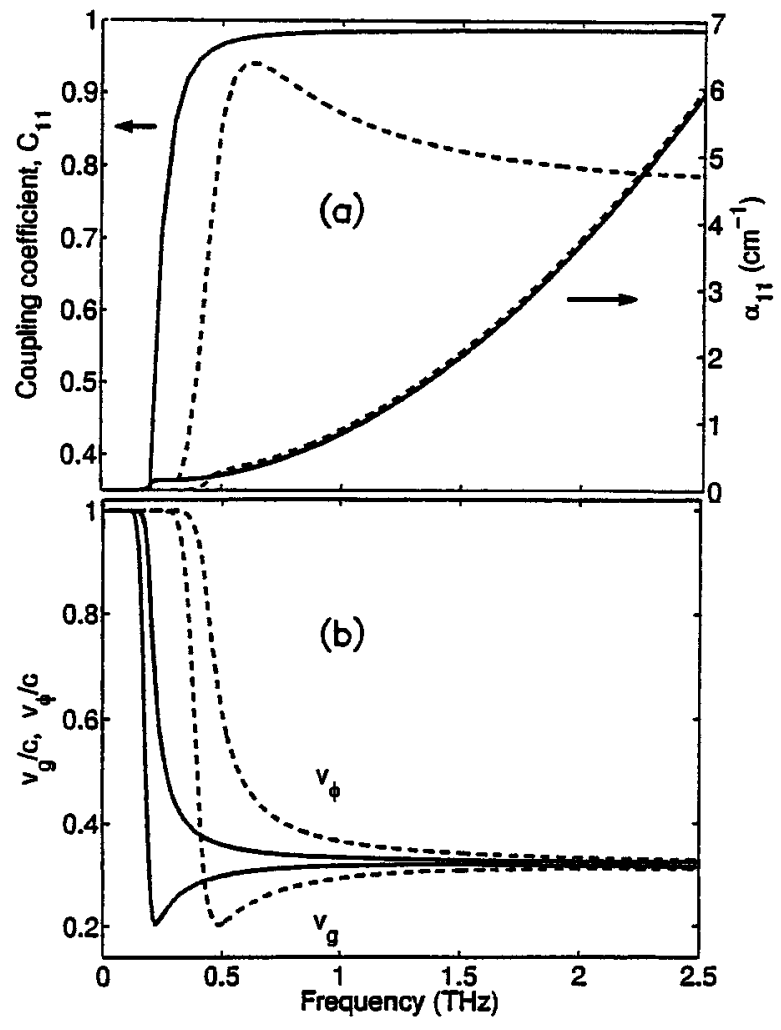

Fig. 19. Properties of the $\mathrm{HE}_{11}$ mode of sapphire fibers of diameter $325 \mu \mathrm{m}$ (solid line) and $150 \mu \mathrm{m}$ (dashed line). (a) The power absorption coefficient (right axis, lower curves) and coupling coefficient (left axis). (b) The calculated group and phase velocities. The upper curves are the phase velocities $v_{\phi}$, while the group velocities $v_{g}$ are given by the lower curves.

in both the time and frequency domains, excellent agreement is obtained between theory and experiment.

A significant experimental and theoretical study of single-mode propagation and quasi-optic coupling of ps $\mathrm{THz}$ pulses into plastic ribbon planar waveguides has just been reported [43]. Dispersive, low-loss propagation was observed within the bandwidth from 0.1 to $3.5 \mathrm{THz}$ for 2-cm-wide ribbon waveguides made of high-density polyethylene, having dimensions of $150 \mathrm{~m}$ (thick) by $10 \mathrm{~mm}$ (long), and $120 \mathrm{~m}$ (thick) by $20 \mathrm{~mm}$ (long). The large group velocity dispersion of the waveguide, opposite to that of hollow metal waveguides and the sapphire fiber waveguide discussed above, caused extensive pulse reshaping and broadening, resulting in positively chirped output pulses. The experiment and calculations based on the well-known 2-D slab waveguide model showed that the linearly polarized (perpendicular to the plane of the ribbon) incoming $\mathrm{THz}$ beam coupled predominantly to the dominant TM mode resulting in single-mode propagation, even though the wideband input spectrum extends beyond the cutoff frequencies of several higher order modes. The experimental setup was similar to that used for the cylindrical and rectangular wave guides shown in Fig. 16(a), except that plano-cylindrical lenses were used.

This study demonstrated the feasibility of the plastic ribbon waveguide as a low-loss, single-mode transmission channel for the terahertz region, capable of utilizing efficient quasi-optic coupling. Ultralow-loss lines are possible by reducing the thickness of the ribbon such that most of the guided wave energy propagates outside the core region. The performance could be 
further enhanced by the use of a very low-loss material such as high-resistivity silicon. Due to the ability to change the group velocity dispersion (GVD) by changing the thickness of the ribbon waveguide, large frequency regions with essentially no GVD can be obtained. In addition, by changing the sign of GVD of the ribbon waveguide with respect to that of the metal and fiber waveguides, mutual pulse compression should be possible, in analogy to dispersion compensation with optical fibers.

\section{SUMMARY AND CONCLUSION}

An overview of experimental efforts to obtain a $\mathrm{THz}$ ultra-wideband transmission channel has been presented. The importance of ultrafast optoelectronics has been paramount in this effort, whereby the combination of optics, ultrafast laser pulses, and electronics has enabled bandwidth and performance increases of as much as 100 times, compared to purely electronic techniques. The importance of complete characterization of transmission lines and waveguides has been illustrated by select examples, where characterization was achieved by THz TDS. For these characterizations, it is imperative that single-mode excitation and propagation are achieved. Sliding contact excitation of coplanar transmission lines was shown to be such a single-mode excitation method. For the $\mathrm{THz}$ waveguide investigations, quasi-optical coupling techniques were shown to be capable of exciting only a single mode of both metal and dielectric waveguides, even though the many $\mathrm{THz}$ bandwidth of the incoming pulse covered as much as 30 waveguide modes.

The performance of lithographically defined planar transmission lines was shown to be limited by the resistive loss of the metal lines, Cherenkov radiation loss from the propagating pulse, and absorption by the underlying dielectric. Typically, for $\mathrm{THz}$ bandwidth pulses, Cherenkov radiation is the dominant loss process, which can be so severe that after propagating only $1 \mathrm{~mm}$, power at $0.8 \mathrm{THz}$ is reduced to $1 / e$ of its original value. Various techniques have been demonstrated to reduce this radiation. The most straightforward approach, which is compatible with VLSI processing, is to reduce line dimensions (linewidths and separations) to below $1 \mu \mathrm{m}$. However, for such dimensions resistive loss of the lines restricts the bandwidth. Normal superconductors can solve this problem for low-frequency applications up to the bandgap frequency of typically $0.7 \mathrm{THz}$, above which the line shows severe loss. As an added benefit, cryogenic temperatures usually lower the $\mathrm{THz}$ absorption loss of crystalline substrates. To achieve high-frequency response with submicrometer linewidths, the high $T_{c}$ superconductors with bandgaps above $15 \mathrm{THz}$ appear as a possible solution. Early attempts to achieve $\mathrm{THz}$ bandwidths with high $T_{c}$ transmission lines were limited by the extremely high absorptive loss of the lattice-matched YSZ substrate. Later investigations used lattice-matched lanthanum aluminate substrates with much lower loss and achieved significantly lower propagation loss up to $1 \mathrm{THz}$ at cryogenic temperatures for a high $T_{c}$ coplanar transmission line compared to an identical transmission line with gold lines. However, a more recent investigation of high $T_{c}$ coplanar transmission lines fabricated on a lattice-matched $\mathrm{MgO}$ substrate found the disappointing result that at $77 \mathrm{~K}$ the attenuation was higher for the high $T_{c}$ line than for an identical gold line. This situation must be resolved, if high $T_{c}$ lines are going to realize their promise as the future material for the VLSI interconnect with dimensions below $1 \mu \mathrm{m}$ and with $\mathrm{THz}$ bandwidths.

$\mathrm{THz}$ waveguides have been recently demonstrated to be an alternative to transmission lines. The attenuation of metal waveguides has been shown to be more than 10 times lower than that for lithographically defined transmission lines on dielectric substrates. In addition, bandwidths up to $3.5 \mathrm{THz}$ have been transmitted with minimum loss. Within the passband of the waveguide, the measured power coupling into the waveguide was typically $40 \%$ of the incoming $\mathrm{THz}$ power. Dielectric waveguides do not have the sharp low-frequency cutoff of metal waveguides and thereby extend the low-frequency limit. The initial demonstration used single-crystal sapphire fibers and achieved single $\mathrm{HE}_{11}$ mode propagation. The waveguide absorption was that of bulk sapphire. A later demonstration achieved single mode propagation in a planar waveguide in the form of a thin ribbon of plastic ribbon. Given a suitable low-loss dielectric, such as high-resistivity silicon with a power absorption coefficient of less than $0.05 \mathrm{~cm}^{-1}$, dielectric waveguides could have much less absorption than metal waveguides. Such dielectric single-mode $\mathrm{THz}$ waveguides would have the promise of an extremely low-loss, flexible interconnect and communications channel, with advantages similar to those of single-mode optical fiber or simple plastic ribbons. Because the group velocity dispersion of the planar waveguides can be opposite to that of the metal tube and fiber waveguides, dispersion compensation or mutual pulse compression between the two type waveguides should be possible.

\section{ACKNOWLEDGMENT}

It has been the author's pleasure to have worked with the following outstanding scientists for various lengths of time, while we developed and demonstrated the usefulness of the optoelectronic techniques applied to single-mode coplanar transmission lines, the THz-TDS system, and the THz waveguides described in this review. The individuals are: J. C. Chen, R. A. Cheville, J. C. C. Chi, F. E. Doany, I. N. Duling, III, C. Fattinger, B. N. Flanders, W. J. Gallagher, G. Gallot, R. H. M. Groeneveld, N. J. Halas, J.-M. Halbout, H. Harde, S. Jamison, T.-I. Jeon, N. Katzenellenbogen, S. R. Keiding, M. B. Ketchen, R. W. McGowen, R. Mendis, R. Menon, J. A. Misewich, S. E. Ralph, N. F. Scherer, A. G. Schrott, and M. van Exter. H. Chan provided most of the excellent masks and wafer fabrication. It is the author's honor to describe their accomplishments.

\section{REFERENCES}

[1] D. H. Auston, "Ultrafast optoelectronics," in Ultrashort Laser Pulses and Applications, (Topics in Applied Physics), 1st ed, W. Kaiser, Ed. Berlin, Germany: Springer-Verlag, 1988, pp. 183-233.

[2] J. A. Valdmanis and G. A. Mourou, "Subpicosecond electro-optic sampling: Principles and applications," IEEE J. Quantum Electron., vol. QE-22, pp. 69-78, Jan. 1986.

[3] D. E. Cooper, "Picosecond optoelectronic measurement of microstrip dispersion," Appl. Phys. Lett., vol. 47, pp. 33-35, 1985.

[4] M. B. Ketchen, D. Grischkowsky, T. C. Chen, C.-C. Chi, I. N. Duling, III, N. J. Halas, J.-M. Halbout, J. A. Kash, and G. P. Li, "Generation of sub-picosecond electrical pulses on coplanar transmission lines," Appl. Phys. Lett., vol. 48, pp. 751-753, 1986.

[5] D. Grischkowsky, M. B. Ketchen, C.-C. Chi, I. N. Duling, III, N. J. Halas, J.-M. Halbout, and P. G. May, "Capacitance free generation and detection of sub-picosecond electrical pulses on coplanar transmission lines," IEEE J. Quantum Electron., vol. 24, pp. 221-225, Feb. 1988. 
[6] J. M. Wiesenfeld, "Electro-optic sampling of high-speed devices and integrated circuits," IBM J. Res. Develop., vol. 34, pp. 141-161, 1990.

[7] D. Grischkowsky, S. Keiding, M. van Exter, and C. Fattinger, "Far-infrared time-domain spectroscopy with TeraHz beams of dielectrics and semiconductors," J. Opt. Soc. Amer. B, vol. 7, pp. 2006-2015, 1990.

[8] D. Grischkowsky, "An ultrafast optoelectronic THz beam system; Applications to time-domain spectroscopy," Opt. Photonics News, vol. 3 , pp. 21-28, 1992.

[9] M. C. Nuss and J. Orenstein, "Terahertz time-domain spectroscopy," in Millimeter and Submillimeter Wave Spectroscopy of Solids, G. Gruener, Ed. Berlin, Germany: Springer-Verlag, 1998, pp. 7-50. (Topics in Applied Physics, No. 74).

[10] M. van Exter and D. Grischkowsky, "Characterization of an optoelectronic TeraHz beam system," IEEE Trans. Microwave Theory Tech., vol. 38, pp. 1684-1692, Nov. 1990.

[11] R. W. McGowan, G. Gallot, and D. Grischkowsky, "Propagation of ultrawideband short pulses of terahertz radiation through submillimeter-diameter circular waveguides," Opt. Lett., vol. 24, pp. 1431-1433, 1999.

[12] G. Gallot, S. Jamison, R. W. McGowan, and D. Grischkowsky, "THz waveguides," J. Opt. Soc. Amer. B., Opt. Phys., vol. 17, pp. 851-863, 2000.

[13] S. P. Jamison, R. W. McGowan, and D. Grischkowsky, "Single-mode waveguide propagation and reshaping of sub-ps terahertz pulses in sapphire fibers," Appl. Phys. Lett., vol. 76, pp. 1987-1989, 2000.

[14] F. E. Doany, D. Grischkowsky, and C.-C. Chi, "Carrier lifetime vs ionimplantation dose in silicon on sapphire," Appl. Phys. Lett., vol. 50, pp. 460-462, 1987.

[15] K. C. Gupta, R. Garg, and I. J. Bahl, Microstrip Lines and Slotlines. Norwood, MA: Artech House.

[16] N. J. Halas, I. N. Duling, III, M. B. Ketchen, and D. Grischkowsky, Proc. Conf. Lasers and Electro-Optics, 1986, OSA Tech. Dig. Ser. Washington, DC: Optical Society of America, 1986, pp. 328-329.

[17] D. Grischkowsky and S. Keiding, "THz time-domain spectroscopy of high $T_{c}$ substrates," Appl. Phys. Lett., vol. 57, pp. 1055-1057, 1990.

[18] S. Ramo, J. R. Whinnery, and T. van Duzer, Fields and Waves in Communication Electronics, 3rd ed. New York: Wiley, 1994.

[19] W. J. Gallagher, C.-C. Chi, I. N. Duling, III, D. Grischkowsky, N. J. Halas, M. B. Ketchen, and A. W. Kleinsasser, "Subpicosecond optoelectronic study of resistive and superconductive transmission lines," Appl. Phys. Lett., vol. 50, pp. 350-352, 1987.

[20] D. Grischkowsky, R. Sprik, M. B. Ketchen, C.-C. Chi, I. N. Duling, III, P. Chaudhari, R. H. Koch, and R. B. Laibowitz, Proc. Conf. Lasers and Electro-Optics, 1988 Tech. Dig. Washington, DC: Optical Society of America, 1988. paper THR2.

[21] D. R. Dykaar, R. Sobolewski, J. M. Chwalek, J. F. Whitaker, T. Y. Hsiang, G. A. Mourou, D. K. Lathrop, S. E. Russek, and R. A. Buhrman, "High-frequency characterization of thin-film Y-Ba-Cu oxide superconducting transmission lines," Appl. Phys. Lett., vol. 52 pp. 1444-1446, 1988.

[22] M. C. Nuss, P. M. Mankiewich, R. E. Howard, B. L. Straughn, T. E Harvey, C. D. Brandle, G. W. Berkstresser, K. W. Goossen, and P. R. Smith, "Propagation of terahertz bandwidth electrical pulses on $\mathrm{YBa}_{2} \mathrm{Cu}_{3} \mathrm{O}_{7-\varepsilon}$ transmission lines on lanthanum aluminate," Appl. Phys. Lett., vol. 54, pp. 2265-2267, 1989.

[23] C. J. Osbahr, B. H. Larsen, T. Hoist, Y. Shen, and S. R. Keiding, " $2 \mathrm{THz}$ bandwidth electrical pulses on $\mathrm{Au}$ and $\mathrm{Yba}_{2} \mathrm{Cu}_{3} \mathrm{O}_{x}$ transmission lines," Appl. Phys. Lett., vol. 74, pp. 1892-1894, 1999.

[24] D. Grischkowsky, I. N. Duling, III, J. C. Chen, and C.-C. Chi, "Electromagnetic shock waves from transmission lines," Phys. Rev. Lett., vol. 59, pp. 1663-1666, 1987.

[25] J. V. Jelley, Cerenkov Radiation and Its Applications. New York: Pergamon, 1958

[26] D. B. Rutledge, D. P. Neikirk, and D. P. Kasilingham, "Integrated circuit antennas," in Infrared and Millimeter Waves, K. J. Button, Ed. New York: Academic, 1983, pt. II, vol. 10.

[27] C. Fattinger and D. Grischkowsky, "Observation of electromagnetic shock waves from propagating surface-dipole distributions," Phys. Rev. Lett., vol. 62, pp. 2961-2964, 1989.

[28] - "A Cherenkov source for freely propagating TeraHz beams," IEEE J. Quantum Electron., vol. 25, no. 12, pp. 2608-2610, Dec. 1989.

[29] D. S. Phatak and A. P. Defonzo, "Dispersion characteristics of optically excited coplanar strip lines: Pulse propagation," IEEE Trans. Microwave Theory Tech., vol. 38, no. 5, pp. 654-661, May 1990.

[30] M. Y. Frankel, S. Gupta, J. A. Valdmanis, and G. A. Mourou, "Terahertz attenuation and dispersion characteristics of coplanar transmission lines," IEEE Trans. Microwave Theory Tech., vol. 39, no. 6, pp. 910-916, June 1991

[31] U. D. Keil, D. R. Dykaar, A. F. J. Levi, R. F. Kopf, L. N. Pfeiffer, S. B. Darack, and K. W. West, "High-speed coplanar transmission lines," IEEE J. Quantum Electron., vol. 28, no. 10, pp. 2333-2342, Oct. 1992.
[32] H. Roskos, M. C. Nuss, K. W. Goossen, D. W. Kisker, A. E. White, K T. Short, D. C. Jacobson, and J. M. Poate, "Propagation of picosecond electrical pulses on a silicon-based microstrip line with buried cobalt silicide ground plane," Appl. Phys. Lett., vol. 58, pp. 2604-2606, 1991.

[33] J.-M. Heiliger, M. Nagel, H. G. Roskos, H. Kurz, F. Schnieder, W. Heinrich, R. Hey, and K. Ploog, "Low-dispersion thin-film microstrip lines with cyclotene (benzocyclobutene) as dielectric medium," Appl. Phys. Lett., vol. 70, pp. 2233-2235, 1997.

[34] D. R. Dykaar, A. F. J. Levi, and M. Anzlowar, "Ultrafast coplanar airtransmission lines," Appl. Phys. Lett., vol. 57, pp. 1123-1125, 1990.

[35] W. H. Knox, J. E. Henry, K. W. Goosen, K. D. Li, B. Tell, D. A. B Miller, D. S. Chemla, A. C. Gossard, J. English, and S. Schmitt-Rink, "Femtosecond excitonic optoelectronics," IEEE J. Quantum Electron. vol. 25 , no. 12, pp. 2586-2595, Dec. 1989.

[36] M. Y. Frankel, R. H. Voelker, and J. N. Hilfiker, "Coplanar transmission lines on thin substrates for high-speed, low-loss propagation," IEEE Trans. Microwave Theory Tech., vol. 42, no. 3, pp. 396-402, Mar. 1994.

[37] H.-J. Cheng, J. F. Whitaker, T. M. Weller, and L. P. B. Katehi, "Terahertz-bandwidth characteristics of coplanar transmission lines on low permittivity substrates," Trans. Microwave Theory Tech., vol. 42, no. 12, pp. 2399-2406, Dec. 1994.

[38] R. W. McGowan, D. Grischkowsky, and J. A. Misewich, "Demonstrated low radiative loss of a quadrupole ultrashort electrical pulse propagated on a three strip coplanar transmission line," Appl. Phys. Lett., vol. 71, pp. 2842-2844, 1997.

[39] J. C. Slater, "Microwave electronics," Rev. Mod. Phys., vol. 18, pp. $441-512,1946$.

[40] N. Marcuvitz, Waveguide Handbook. London, U.K.: (Peregrinus on behalf of The Institution of Electrical Engineers), 1986.

[41] A. W. Snyder, "Excitation and scattering of modes on a dielectric or optical fiber," IEEE Trans. Microwave Theory Tech., vol. 17, pp. $1138-1144,1969$

[42] D. R. Dykaar, R. Sobolewski, J. M. Chwalek, J. F. Whitaker, T. Y. Hsiang, G. A. Mourou, D. K. Lathrop, S. E. Russek, and R. A. Buhrman, Erratum to, "High-frequency characterization of thin-film Y-Ba-Cu oxide superconducting transmission lines," Appl. Phys. Lett., vol. 52, p. 2003, 1988.

[43] R. Mendis and D. Grischkowsky, "Plastic ribbon THz waveguides," $J$. Appl. Phys., vol. 88, pp. 4449-4451, 2000.

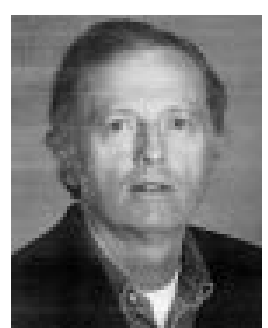

Daniel R. Grischkowsky (A'84-SM'90-F'92) was born in St. Helens, OR, on April 17, 1940. He received a bachelor's degree from Oregon State University in 1962 and the Ph.D. degree in physics from Columbia University in 1968. His thesis work, supervised by S. R. Hartmann, involved electron spin resonance investigations and led to the explanation of the magnetic field dependence of photon echoes in ruby.

In 1969, he joined IBM at the Watson Research Center at Yorktown Heights, NY. His initial experimental and theoretical research involved studying the interaction between near-resonant light and the two-level system. The adiabatic following model, which he originally proposed as a result of these studies, explained the observed effects of self-focusing, self-defocusing, self-steepening, and slow group velocities in vapors of alkali metals. His subsequent experimental and theoretical studies of the nonlinear propagation of picosecond laser pulses in single-mode optical fibers led to the invention of the optical-fiber pulse compressor and to the experimental observations of gray solitons and optical intensity shocks. He has since shifted his work to ultrafast optoelectronics, initially focusing on the generation and applications of subpicosecond electrical pulses on transmission lines. An important part of this work has been the observation of terahertz $(\mathrm{THz})$ radiation from the generation site and Cerenkov radiation from the propagating electrical pulses. These studies have resulted in new sources of pulsed $\mathrm{THz}$ beams, which have enabled the new technique of $\mathrm{THz}$ time-domain spectroscopy (THz-TDS). In 1993, he relocated to Oklahoma State University as the Bellmon Professor of Optoelectronics in the School of Electrical and Computer Engineering. At OSU, his work has concentrated on unique applications of THz-TDS, including determining the nature of conductivity in semiconductors, terahertz characterizations of transmission lines and waveguides, and terahertz impulse scale ranging.

Dr. Grischkowsky is a fellow of the Optical Society of America and the American Physical Society. He was awarded the Boris Pregel Award for Applied Science and Technology (1985) by the New York Academy of Sciences for his invention of the optical fiber pulse compressor. He received the R. W. Wood Prize (1989) from the Optical Society of America for his pulse propagation studies in optical fibers and their use for generating ultrashort pulses of light. 